\title{
In Situ Optical Quantification of Extracellular Electron Transfer using Plasmonic Metal Oxide Nanocrystals
}

Austin J. Graham ${ }^{* 1,2}$, Stephen L. Gibbs ${ }^{* 1}$, Camila A. Saez Cabezas ${ }^{* 1,2}$, Yongdan Wang ${ }^{1}$, Allison M. Green ${ }^{1,2}$, Delia J. Milliron^1,2 , Benjamin K. Keitz ${ }^{\wedge 1,2}$

${ }^{1}$ McKetta Department of Chemical Engineering, University of Texas at Austin, Austin, TX 78712

${ }^{2}$ Center for Dynamics and Control of Materials, University of Texas at Austin, Austin, TX 78712

${ }^{*}$ These authors contributed equally.

^To whom correspondence should be addressed: milliron@che.utexas.edu; keitz@utexas.edu

\section{Abstract}

Extracellular electron transfer (EET) is a critical form of microbial metabolism that enables respiration on a variety of inorganic substrates, including metal oxides. However, quantifying current generated by electroactive bacteria has been predominately limited to biofilms formed on electrodes. To address this, we developed a platform for quantifying EET flux from cell suspensions using aqueous dispersions of infrared plasmonic tin-doped indium oxide nanocrystals. Tracking the change in optical extinction during electron transfer enabled quantification of current generated by planktonic Shewanella oneidensis cultures. Using this method, we differentiated between starved and actively respiring cells, between cells of varying genotype, and between cells engineered to differentially express a key EET gene using an inducible genetic circuit. Overall, our results validate the utility of colloidally stable plasmonic metal oxide nanocrystals as quantitative biosensors in native biological environments and contribute to a fundamental understanding of planktonic S. oneidensis electrophysiology using simple in situ spectroscopy.

\section{Introduction}

Extracellular electron transfer (EET) is a microbial respiratory process by which electron flux from carbon metabolism is transported across the cell membrane and directed onto inorganic substrates (1). This capability makes electrogenic bacteria useful organisms for microbial fuel cells $(2,3)$, bioelectronics $(4-6)$, material syntheses $(7,8)$, and has led to significant interest in controlling their electron flux using synthetic biology $(9,10)$. Improving EET-dependent technologies such as controlling electron transfer dynamics (11), implementing genetic logic (12), and orchestrating electron transfer dynamics between proteins (13) necessitates a stronger quantitative understanding of bacterial electrophysiology and simple methods to measure current generation. EET is typically quantified from biofilms formed on electrodes in microbial fuel cells (MFCs) or bioelectrochemical systems (BESs) (1, 14-16). EET from single cells has also been measured in such systems, providing sensitive quantitative measurements and highlighting 
38 variability in EET capabilities between individual cells (17-19). However, these measurements

39 often require long incubation times and electrode colonization ( days), and the contribution of

40 suspended (i.e., planktonic) microbes is generally unclear. Furthermore, implementation of

41 synthetic gene programs such as genetic logic operations often require cell turnover and growth,

42 which are more complex in biofilms compared to planktonic culture. While there has been recent

43 progress toward quantifying EET from planktonic cells in BESs (20), EET from planktonic

44 microbes is more traditionally quantified optically, using soluble substrates such as dye-bound

45 iron citrate or riboflavin $(12,21-23)$. We propose that by expanding upon the optical analysis

46 toolkit by employing dispersed, plasmonic nanoparticles, we will enable greater understanding of

47 EET to insoluble substrates.

49 Plasmonic nanoparticles, though insoluble, maintain colloidal stability in a variety of solvents. An 50 intrinsically high concentration of conductive electrons results in a strong absorption at the 51 localized surface plasmon resonance (LSPR) peak frequency, $\omega_{L S P R}$, and enables participation

52 in redox reactions with molecules near the surface many times over. The LSPR peak position and 53 intensity depend on the particle's conduction electron concentration, $n_{e}$, such that tracking the 54 optical response near $\omega_{L S P R}$ can report on the number of redox reactions occurring at the 55 nanoparticle surface (24-26). Thus, optically tracking the plasmonic response can monitor 56 electron transfer similar to tracking current from a traditional film electrode. Doped metal oxide 57 nanocrystals are particularly promising candidates because they sustain plasmonic resonance 58 but have $n_{e} \sim 1-3$ orders of magnitude lower than $\mathrm{Au}$ and $\mathrm{Ag}(27,28)$, making their optical 59 response more sensitive to single electron transfer events and also placing $\omega_{L S P R}$ in the infrared $60(24,25)$.

62 Doped metal oxide nanocrystals have a few advantages over traditional optically-monitored 63 acceptors such as iron citrate and flavins. First, $\omega_{L S P R}$ can be tuned throughout the near- to mid64 infrared. This enables tailoring the peak absorption to the ideal window for each experimental 65 setting, and leaves the visible spectrum available for separate and simultaneous measurements. 66 Further, the surface of doped metal oxide nanocrystals can be post-synthetically functionalized 67 with various ligands to customize solvent compatibility and tune the interactions with other species 68 in solution (29-31). In addition, a large insoluble acceptor should inhibit passive and active 69 transport across the cell membrane (32), both of which could convolute reduction pathways when 70 using small molecule acceptors such as iron citrate or flavins. For example, microbial iron 
71 reduction does not necessarily translate to electricity generation on an electrode from that

72 organism (33).

74 Overall, there is a need for reliable in situ biosensors of EET that are compatible with planktonic 75 culture. Indeed, the advantageous properties of metal oxide nanocrystals have already enabled 76 their use in spectroscopic measurement of EET (34-37). However, these methods did not directly 77 quantify the relationship between the spectroscopic response and the electron transfer rate (i.e., 78 current generation). We recently developed a model for fitting the plasmonic optical response of 79 doped metal oxide nanocrystal dispersions which extracts the number of free electrons in each 80 nanocrystal, enabling in situ tracking of biological electron transfer events using infrared 81 spectroscopy (38).

83 Leveraging our ability to quantify the change in optical response with the aqueous biocompatibility 84 of colloidal nanocrystal dispersions, we aimed to quantify in situ electron flux from planktonic 85 microbes in culture suspensions. We chose Shewanella oneidensis as a model electroactive 86 bacterium that is attractive for its respiratory plasticity and genetic tractability. Using nondestructive optical measurements to track the LSPR peak in real-time, we successfully quantified kinetic EET rate constants from $S$. oneidensis of varying metabolic activity and genotype. We also quantitatively differentiated electron flux from an engineered $S$. oneidensis strain with tuned EET gene expression levels. Our method enabled quantification of $S$. oneidensis population currents

91 from planktonic culture, with the average cell exhibiting $0.05-2.8 \mathrm{fA}^{-}$cell $^{-1}$ depending on

92 metabolic activity, genotype, and gene expression level. Overall, our results indicate that 93 plasmonic semiconductor nanocrystals are a reliable infrared sensing platform for probing 94 metabolic activity in cellular environments and contribute to a quantitative understanding of EET.

\section{Results \& Discussion}

\section{Preparation of Sn-doped indium oxide (ITO) nanocrystal aqueous dispersions.}

ITO nanocrystals (5.78 $\pm 0.64 \mathrm{~nm}$ radius, 9.4 at\% $\mathrm{Sn}$ ) were synthesized using established colloidal

101 methods and a slow injection approach (Figure 1a, Materials and Methods) (39). Nanocrystal size

102 and Sn doping concentration were measured with small-angle X-ray scattering (SAXS) (Figure

103 S1) and inductively coupled plasma-optical emission spectroscopy, respectively. To disperse ITO 104 nanocrystals in aqueous solvents, the hydrophobic organic ligands bound to the surface of the 
as-synthesized nanocrystals were replaced by a hydrophilic polymer. Specifically, we chose poly(acrylic acid) grafted with methoxy-terminated poly(ethylene oxide) (PAA-mPEO 4 ). This custom-designed and biocompatible random co-polymer has previously been used to functionalize different types of metal oxides $(40,41)$ and been shown to be suitable for facilitating electrochemical redox reactions in thin films of polymer-wrapped ITO nanocrystals (42).

a

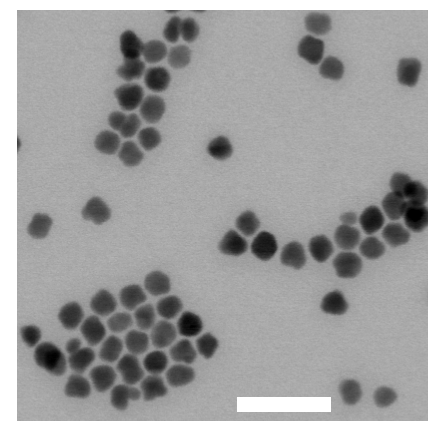

b

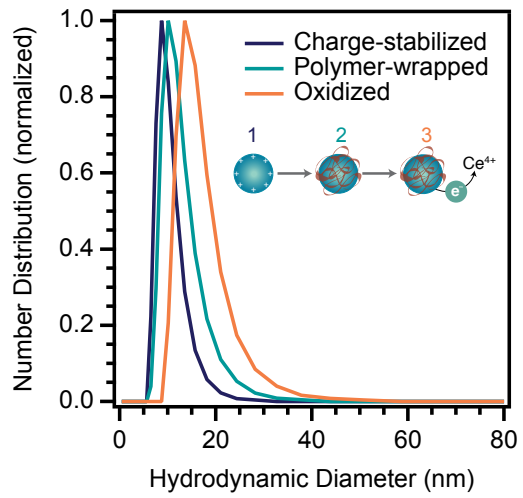

C

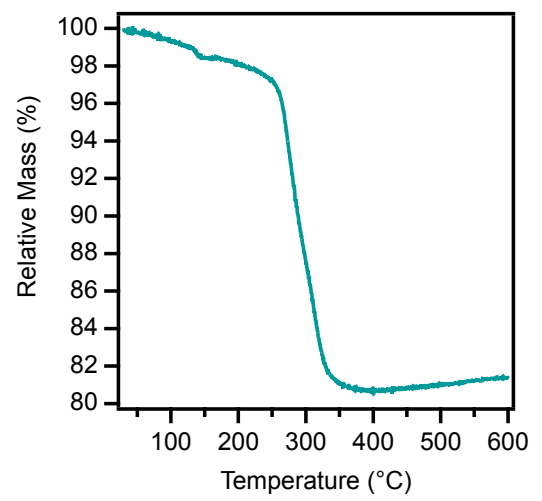

Figure 1. Preparation of polymer-wrapped oxidized nanocrystals for EET biosensing. (a) Scanning transmission electron microscopy image of as-synthesized ITO nanocrystals. Scale bar $=40 \mathrm{~nm}$. (b) Dynamic light scattering of charge-stabilized, polymer-wrapped, and oxidized polymer-wrapped ITO nanocrystals. The ITO nanocrystals remain colloidally stable and do not exhibit signs of significant aggregation after each processing step (inset) since the hydrodynamic diameter measurements of the three samples are in close agreement. (c) Quantification of polymer adsorption on the ITO nanocrystal surface using thermogravimetric analysis. The mass loss $(18 \%)$ around $300{ }^{\circ} \mathrm{C}$ corresponds to the decomposition of PAA-mPEO 4.

The functionalization of ITO nanocrystals with $\mathrm{PAA}^{-m \mathrm{PEO}_{4}}$ was achieved in a two-step process. First, the native stabilizing ligands were chemically removed by exposing the ITO nanocrystals to nitrosonium tetrafluoroborate, which resulted in bare, charge-stabilized ITO nanocrystals dispersible in $\mathrm{N}, \mathrm{N}$-dimethylformamide (31). Then, charge-stabilized ITO nanocrystals were mixed with PAA-mPEO ${ }_{4}$ and subsequently transferred into Milli-Q water. In a previous study, we found that colloidal stability in aqueous media is due to the adsorption of PAA-mPEO 4 on ITO and is likely facilitated by the coordination of carboxylate species at the nanocrystal surface (42). Fourier transform infrared spectroscopy of nanocrystal dispersions at each processing step confirmed that the native ligands were effectively removed and replaced by PAA-mPEO (Figure S2). Finally, polymer-wrapped ITO nanocrystals were chemically oxidized upon the addition of ammonium cerium (IV) nitrate to promote EET from S. oneidensis. As expected, oxidation of the nanocrystal dispersion caused a red-shift and decrease in intensity of the LSPR peak (Figure S3a). After oxidation, the polymer-wrapped ITO nanocrystals were dispersed in a deuterated medium (Shewanella Basal Medium, SBM, Table S1). Deuterated buffer avoided saturating the spectrophotometer detector, as strong water absorption in the near-infrared (NIR) (43) overlaps 
135 significantly with the ITO LSPR peak (Note S1). A non-deuterated buffer could in principle be

136 used by employing a doped metal oxide nanocrystal material with higher energy $\omega_{L S P R}$, such as

137 F, In co-doped CdO (27).

139 Avoiding the formation of large ITO nanocrystal aggregates in the dispersion before or during EET

140 is key for interpretation and analysis of optical measurements. Therefore, we monitored the 141 hydrodynamic diameter of the nanocrystal dispersion after each processing step (i.e., ligand 142 removal, polymer wrapping, and oxidation post-polymer wrapping) using dynamic light scattering 143 to probe the stability of the colloid (Figure 1b). The hydrodynamic diameters of the charge144 stabilized and polymer-wrapped samples were centered around $\sim 10 \mathrm{~nm}$, which is in good 145 agreement with the diameter obtained from SAXS. The slight increase in the hydrodynamic 146 diameter of the polymer-wrapped and oxidized sample $\left(D_{h} \sim 15 \mathrm{~nm}\right)$ could suggest interaction 147 between the colloid and charged species during oxidation, but we did not detect signs of 148 significant aggregation. This dispersion was colloidally stable for at least five months after 149 oxidation and transfer into deuterated SBM (Figure S3b). We hypothesize that the colloidal 150 stability of our aqueous dispersions of polymer-wrapped ITO (PAAPEO-ITO) is due to sufficient 151 PAA-mPEO 4 adsorption on the nanocrystal surface (18\% by mass, Figure 1c), which we 152 quantified by thermogravimetric analysis, and thus effective steric stabilization.

\section{S. oneidensis remains viable in the presence of PAAPEO-ITO.}

All bacterial strains and plasmids used in this study are listed in Table S3. We first ensured that 157 polymer-wrapped nanocrystals were not cytotoxic by quantifying cell viability in their presence 158 (44). Aerobically pregrown cells were diluted to $\mathrm{OD}_{600}=0.2$ in a mixture of $\sim 1 \mathrm{mg} / \mathrm{mL}(\sim 0.2 \mu \mathrm{M})$ 159 PAAPEO-ITO and aerobically incubated for $2 \mathrm{~h}$ at $30^{\circ} \mathrm{C}$. Cells were then stained for membrane 160 permeability using the BacLight Live/Dead kit (Invitrogen) according to manufacturer protocols. 161 Unbound Live/Dead stain was washed away and cells were imaged using green (live) and red 162 (dead) fluorescence channels. Under these conditions, S. oneidensis MR-1 (wild-type) remained 163 predominately alive, with an $86 \pm 2 \%$ viable population (Figure S4). To assess cell health for 164 longer experiments, colony counting was used to quantify the viable population after $8 \mathrm{~h}$ 165 incubation with PAAPEO-ITO and $20 \mathrm{mM}$ lactate at room temperature in deuterated SBM with 166 casamino acids. As expected, there were not significant effects on the viable population (Figure 167 S5). Minor decreases in viable population can likely be attributed to the lack of electron acceptor 168 aside from PAAPEO-ITO, which was present at $\sim 1.5 \mathrm{mg} / \mathrm{mL}(\sim 0.3 \mu \mathrm{M})$ for EET quantification 
169 experiments (compared to standard growth conditions in $40 \mathrm{mM}$ fumarate). In contrast to

170 fumarate, a nanocrystal can accept more than one electron, but not enough to account for this

171 concentration difference. These results indicate that cell viability is not substantially impacted

172 beyond electron acceptor limitation, and that polymer-wrapped nanocrystals are not cytotoxic to

173 S. oneidensis.

174

175 Optical extinction of PAAPEO-ITO tracks respiratory electron flux from S. oneidensis.

177 Once cell viability was established, we measured the in situ optical response of the PAAPEO-ITO

178 LSPR during electron transfer from S. oneidensis MR-1. Aerobically pregrown cells were washed

179 and resuspended in $1.5 \mathrm{mg} / \mathrm{mL}$ PAAPEO-ITO in deuterated SBM with casamino acids and 20

$180 \mathrm{mM}$ lactate as the carbon source. The solution was immediately transferred to a cuvette, sealed,

181 and loaded into a spectrophotometer in transmission mode to monitor the LSPR peak over time.

182 The time-resolved increase in optical density and blue shift of the LSPR peak results from an 183 increasing number of electrons within the ITO NCs, which indicates continuous electron transfer 184 to the nanocrystals (Figure 2a). These results confirm that $S$. oneidensis is able to reduce 185 PAAPEO-ITO and the kinetics of this process are resolvable using NIR spectroscopy. Given the 186 aerobic preparation of the cell culture and sample, they also suggest that dissolved oxygen is first 187 consumed through aerobic respiration, followed by activation of anaerobic EET pathways (i.e., 188 reduction of PAAPEO-ITO), as we have previously observed for other electron acceptors such as 189 copper and fumarate $(45,46)$. We then measured EET from a S. oneidensis MR-1 population 190 deprived of a carbon source (e.g., lactate), which should inhibit respiration and prevent generation 191 of electron flux. The change in optical extinction was significantly diminished in these samples 192 (Figure 2b). Some minimal reduction was observed, which can likely be attributed to excess 193 metabolism from pregrowth (7), or to non-specific reducing capacity from cell stress and lysis. 194 Indeed, a reaction containing lysed S. oneidensis slightly reduced PAAPEO-ITO even without 195 oxygen removal, indicating that cell death could lead to a small amount of nonspecific reduction 196 (Figure S6). Similarly, an E. coli sample demonstrated minimal PAAPEO-ITO reduction, as would 197 be expected from this EET-deficient microbe (Figure 3c). Together, these measurements confirm 198 that regulating metabolic flux manifests in an observable difference to the optical response of 199 PAAPEO-ITO, validating this platform as an effective in situ biosensor that is specific to EET. 


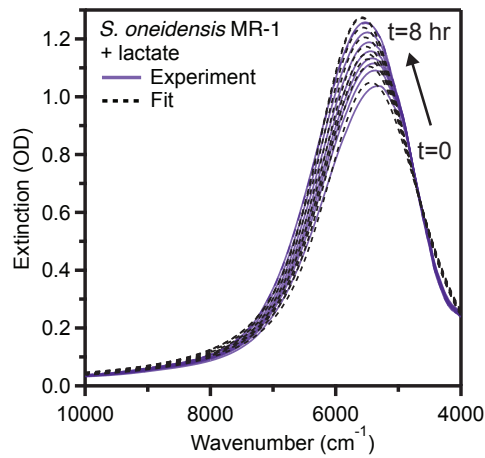
with $20 \mathrm{mM}$ lactate as a carbon source. an infrared sensing platform. b

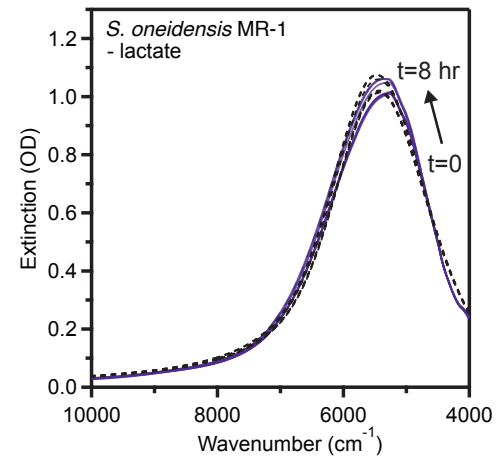

C

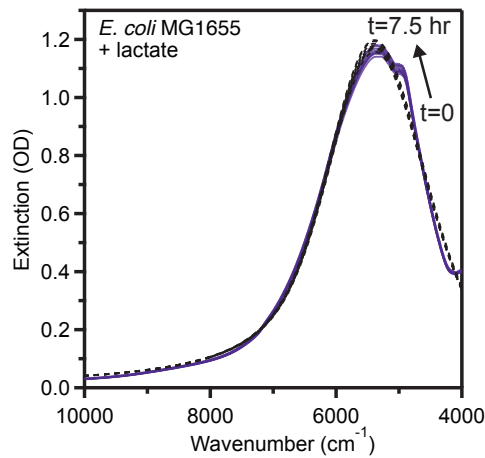

Figure 2. EET from metabolically active $S$. oneidensis leads to time-resolved increased optical extinction of PAAPEO-ITO. Experimental extinction spectra collected each hour from $t=0$ to $8 \mathrm{~h}$ and associated fits for PAAPEO-ITO inoculated with (a) S. oneidensis MR-1 that was provided $20 \mathrm{mM}$ lactate as a carbon source, (b) S. oneidensis MR-1 not provided a carbon source, or (c) E. coli MG1655 provided

As a proof of concept, we also collected extinction out to higher energies to test if we could simultaneously measure the infrared LSPR spectrum and meaningful data in the visible wavelengths. We chose to quantify the intensity of scattered light by the cells at $600 \mathrm{~nm}\left(\mathrm{OD}_{600}\right)$ because this value is used to quantify the concentration of cells in a dispersion. We were able to successfully monitor both the infrared plasmon peak and the $\mathrm{OD}_{600}$ value in a single sweep of wavelengths in our spectrophotometer (Figure S7), further illustrating the benefits of developing

\section{Quantitative spectral analysis enables quantification of EET from $S$. oneidensis with} varying metabolic activity and genotype.

Following qualitative observations of the LSPR peak over time, we fit the peaks to a free electron model that extracts the number of conductive electrons in a nanoparticle. Using a deuterated buffer allowed for the collection of clean spectra and nearly the full plasmon peak for the ITO nanocrystals could be resolved. ITO nanocrystals were previously incubated with $S$. oneidensis,

228 ITO nanocrystals, our ability to resolve nearly the full plasmonic response allows for a simpler, 229 more reliable quantification using our recently developed model, the heterogeneous ensemble 230 Drude approximation (HEDA). The HEDA model extracts intrinsic material properties, such as 
231 electron concentration, from the plasmonic response of a dilute dispersion of nanocrystals (38).

232 By accounting for ensemble heterogeneities in nanocrystal size and nanocrystal electron

233 concentration, it enables more accurate acquisition of material properties.

Immediately upon inoculating the cells with oxidized PAAPEO-ITO, we began collecting timeresolved spectra, continuing for up to 48 hours. We fit spectra using the HEDA model to quantify

237 the number of electrons within the average nanocrystal in an ensemble at each timepoint (Note

238 S2). Since we quantified both the concentration of nanocrystals and cells, we could calculate the

239 cumulative electrons transferred per cell over time. This quantification revealed two consistent

240 electron transfer regimes across samples - a variable regime during the first $\sim 1 \mathrm{~h}$, where electron

241 transfer was nonlinear (Figure 3a, inset; Figure S8a), followed by a steady-state electron transfer

242 regime. In previous studies, aerobic $S$. oneidensis at $O_{600}=0.2$ took between $10 \mathrm{~min}$ to $1 \mathrm{~h}$ to

243 fully consume dissolved oxygen and turn on anaerobic EET pathways, depending on the electron

244 acceptor and reaction conditions $(45,46)$. Therefore, we attribute this initial variable domain to a

245 combination of oxygen removal via aerobic respiration and cell stress as they adjust to a new

246 electron acceptor and undergo a metabolic shift.

248 Beyond $8 \mathrm{~h}$ of reaction in high EET samples (e.g., wild-type S. oneidensis with lactate), we noticed 249 effects of nanocrystal saturation and precipitation (Figure 3a, Figure S8b). Thus, we defined the 250 steady-state regime as between 1 and $8 \mathrm{~h}$, and used this domain to determine electron transfer 251 kinetics. Notably, $\mathrm{OD}_{600}=0.2$ is approximately the saturation density of anaerobic $S$. oneidensis 252 in SBM with $20 \mathrm{mM}$ lactate, although this varies depending on the electron acceptor. We suspect 253 that upon adjusting to their new anaerobic environment, the cells should divert minimal metabolic 254 flux toward growth and instead direct it toward energy production via EET (47). This, combined 255 with the lack of cell growth between inoculation and $8 \mathrm{~h}$ (Figure S5), validates the appearance of 256 steady-state electron transfer in our system. As such, the linear increase in electrons transferred 257 per cell justified using linear regressions to obtain kinetic rate constants and compute population258 averaged current generation. EET could also be measured in samples containing an added 259 electron acceptor, fumarate. However, to simplify analysis and ensure all metabolic electron flux 260 was directed toward the measured acceptor, PAAPEO-ITO, we chose to omit fumarate from 261 future experiments (Figure S9). 
a

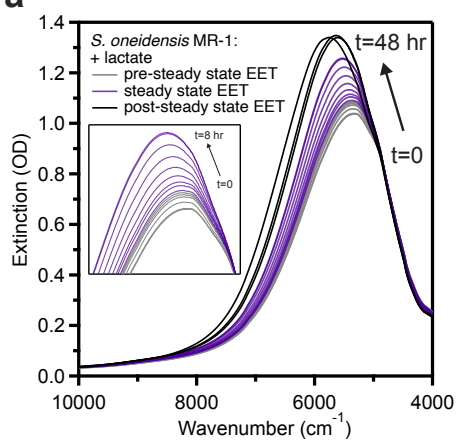

b

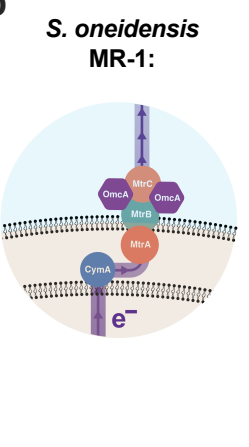

S. oneidensis $\Delta m t r C \Delta$ omcADmtrF:

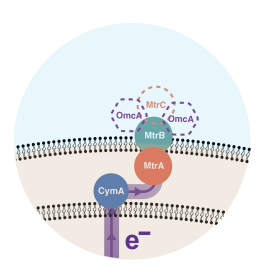

C

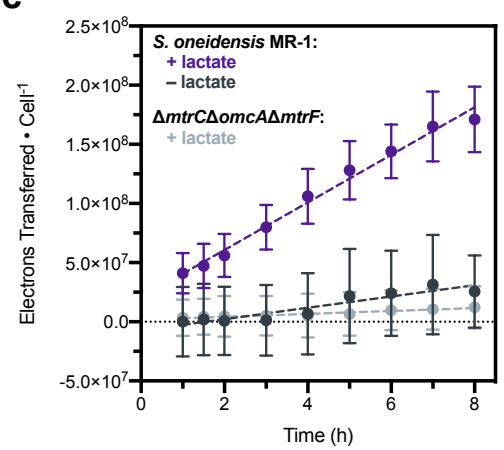

Figure 3. HEDA fitting of PAAPEO-ITO LSPR response during EET from S. oneidensis enables quantification of steady-state electron transfer kinetics. (a) EET from S. oneidensis to PAAPEO-ITO exhibits three domains. (Inset) The early domain $(\leq 1 \mathrm{~h})$ shows variability due to aerobic respiration, introduction of a deuterated solvent, and cell stress. This regime includes a lag phase $(0-20 \mathrm{~min})$ followed by a sharp increase in electron transfer (20-60 min). Steady-state electron transfer (1-8 h) precedes a later domain ( $\geq 8 \mathrm{~h}$ ) exhibiting effects of nanocrystal saturation, precipitation, and cell death. (b) The Mtr pathway in $S$. oneidensis. In the $\Delta m t r C \Delta o m c A \Delta m t r F$ knockout (right), three critical extracellular cytochrome genes are removed from the genome, hindering EET capabilities. (c) Steady-state EET (1-8 h) where dashed lines represent linear regression of the electrons transferred over time and $k_{E E T}$ is the slope of the regression (Table 1). Data points shown are the mean \pm SD of $n=3$ fits of biological replicates.

We first determined steady-state electron transfer rates to PAAPEO-ITO for S. oneidensis MR-1 with and without lactate as a carbon source, which qualitatively showed a large difference in optical response (Figure 2). When $20 \mathrm{mM}$ lactate was present as a carbon source, enabling continuous respiration and generation of EET flux, the rate of electron transfer was $k_{E E T}=2.0 \pm$ $0.2 \times 10^{7}$ electrons $\cdot$ cell $^{-1} \cdot \mathrm{h}^{-1}$, or $0.89 \pm 0.07 \mathrm{fA} \cdot$ cell $^{-1}$, which is approximately 5 -fold greater than when the cells were starved (Table 1). To further assess background electron transfer and dynamic range, we used an EET-deficient strain of $S$. oneidensis. $S$. oneidensis $\Delta m t r C \Delta o m c A \Delta m t r F$ is a triple-knockout of three key extracellular cytochromes responsible for direct electron transfer to a variety of substrates (Figure $3 b$ ), and as a result, exhibits significantly hindered electron transfer activity toward both electrodes and soluble acceptors (11, 14). As expected, this strain showed significantly attenuated electron transfer to PAAPEO-ITO (Figure 3c). Additionally, there was an order-of-magnitude decrease in the electron transfer rate constant compared to the wild-type strain (Table 1). It should be noted that statistical error associated with accumulated electron transfer is dominated by variation in $S$. oneidensis EET rates among biological replicates, not from error associated with spectra collection or fitting (Figure S10). The collected spectra are highly resolved, meaning the signal is stable over the collection time period of $\sim 1$ minute and the fitting procedure consistently matches experimental data and converges to the same solution even for a range of randomly generated initial guesses (Table S6). 
Table 1. Metrics obtained from HEDA fitting during EET from S. oneidensis. Data shown are results of the steady-state regime, between 1 and $8 \mathrm{~h}$, and are the mean \pm SD of $n=3$ HEDA model fits of biological replicates.

\begin{tabular}{|c|c|c|c|}
\hline Sample & $\mathbf{e}^{-}$Transferred $\cdot$ Cell-1 & $\boldsymbol{k}_{\text {EET }}\left(\mathbf{e}^{-} \cdot\right.$ cell-1 $\left.^{-1} \cdot \mathbf{h}^{-1}\right)$ & Current $\cdot$ Cell-1 $(\mathbf{f A})$ \\
\hline $\begin{array}{c}\text { S. oneidensis MR-1 } \\
(+20 \mathrm{mM} \text { lactate })\end{array}$ & $(1.3 \pm 0.2) \times 10^{8}$ & $(2.0 \pm 0.2) \times 10^{7}$ & $0.89 \pm 0.07$ \\
\hline $\begin{array}{c}\text { S. oneidensis MR-1 } \\
(-20 \mathrm{mM} \text { lactate })\end{array}$ & $(2.5 \pm 3.1) \times 10^{7}$ & $(4.8 \pm 2.4) \times 10^{6}$ & $0.21 \pm 0.11$ \\
\hline $\begin{array}{c}\text { S. oneidensis } \\
(+20 \mathrm{mM} \text { lactate })\end{array}$ & $(0.9 \pm 1.4) \times 10^{7}$ & $(1.2 \pm 1.2) \times 10^{6}$ & $0.05 \pm 0.05$ \\
\hline
\end{tabular}

Although the physical dimensions of the nanocrystals $(5.78 \pm 0.64 \mathrm{~nm}$ radius, compared to cell length on the $\sim 1 \mu \mathrm{m}$ scale) should prevent biofilm formation, we sought to ensure that EET was occurring from planktonic cultures. Therefore, we tested a biofilm-deficient knockout ( $S$. oneidensis S2933, termed $\Delta$ lysis-operon) (48). This strain did not exhibit significantly different current generation than wild-type $S$. oneidensis, indicating that electron transfer is occurring from planktonic cells (Figure 4). We repeated the wild-type sample as this experiment was performed with a newly synthesized batch of nanocrystals compared to Figure 3 (Note S3).

a

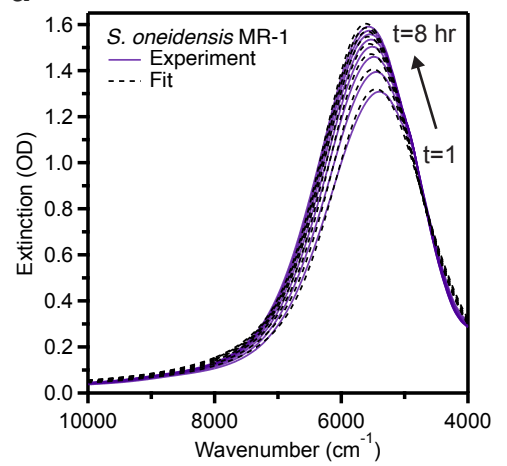

b

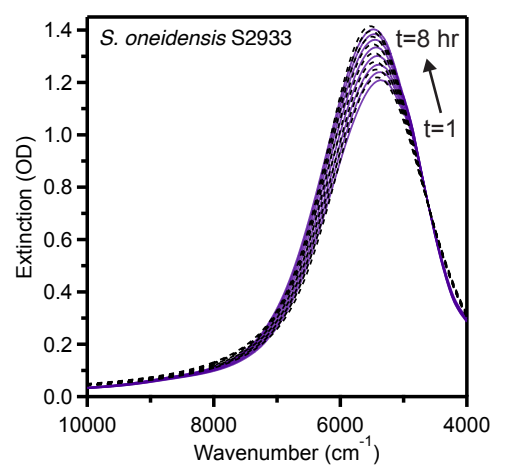

C

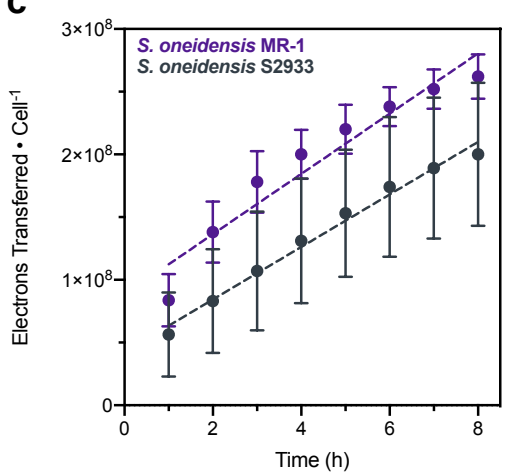

Figure 4. Planktonic cells are responsible for EET to PAAPEO-ITO. Extinction spectra for PAAPEOITO dispersions incubated with (a) S. oneidensis MR-1 and (b) and S. oneidensis S2933 (Alysis-operon, a biofilm-deficient knockout) (48). (c) Steady-state EET (1-8 h) where dashed lines represent linear regression of the electrons transferred over time and $k_{E E T}$ is the slope of the regression (Table 1). For MR$1, k_{E E T}=(2.4 \pm 0.2) \times 10^{7} \mathrm{e}^{-} \cdot$ cell $^{-1} \cdot \mathrm{h}^{-1} ;$ for S2933, $k_{E E T}=(2.1 \pm 0.4) \times 10^{7} \mathrm{e}^{-} \cdot \mathrm{cell}^{-1} \cdot \mathrm{h}^{-1}$. Data points shown are the mean \pm SD of $n=3$ HEDA model fits of biological replicates.

317 All fit results are reported in Tables S4-7. Overall, current quantified from the wild-type strain with

$31820 \mathrm{mM}$ lactate is comparable to, but slightly lower than, results from other methods that have

319 measured or extrapolated single cell electron flux from S. oneidensis to heterogeneous acceptors. 
320 Previous studies found current generation from S. oneidensis can vary, from $\sim 2 \mathrm{fA} \cdot$ cell $^{-1}$ in cell

321 suspensions to soluble riboflavin, to $\sim 100-200 \mathrm{fA} \cdot$ cell $^{-1}$ in single cells to electrodes $(18,23,49)$.

322 Methods measuring larger reduction rates have varied in experimental conditions, including

323 extrapolating single cell EET from biofilms on electrodes or hematite nanoparticles (14, 18-19,

324 49-50). One primary difference in our study compared to these is the lack of cell immobilization

325 on a surface. Cell motility may inhibit certain EET mechanisms such as flavin shuttling, which

326 occurs primarily in attached cytochromes $(19,51)$. Therefore, it is possible that both physiological

327 and experimental differences account for increased EET measurement in these platforms

328 compared to ours. In addition, the necessity of deuterium in our buffer would lead to kinetic isotope

329 effects on proton pumping and therefore electron transfer kinetics. Previous studies have

330 determined that $S$. oneidensis can recover from this and reach deuterium efflux equilibrium (52);

331 however, this may contribute to lower overall current generation measured in our system

332 compared to others (Note S1). Finally, the polymer coating around the nanocrystals could

333 increase the required distance for direct electron transfer, which would also reduce EET rate.

334 Despite these challenges, our results indicate that the decreased rate does not inhibit us from

335 differentiating between the EET rates for strains of varying metabolic activity and genotype.

336 Overall, these results contribute to a quantitative understanding of EET from planktonic cell

337 populations and suggest that simple spectroscopic techniques can be used as a screening tool

338 to elucidate the electroactive capabilities of various species or strains.

EET output from engineered $S$. oneidensis with variable mtrC expression can be quantitatively differentiated using PAAPEO-ITO.

343 Synthetic biologists commonly use genetic circuits to control protein output, and emergent 344 applications of EET will likely employ similar strategies. However, unlike fluorescence or secreted 345 protein, it is difficult to directly quantify the relationship between EET gene expression and 346 resultant electron flux, especially in planktonic cultures. Toward this goal, we used an $S$. 347 oneidensis strain that tailors expression of a critical EET gene, $m t r C$, in response to inducing

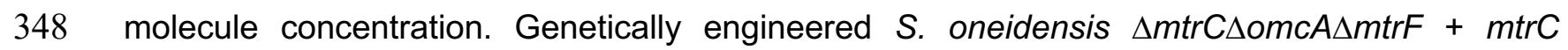
349 proportionally expresses $m t r C$ in the presence of isopropyl ß-D-1-thiogalactopyranoside (IPTG) 350 as an inducer $(12,46)$. Cultures of $S$. oneidensis $\Delta m t r C \Delta o m c A \Delta m t r F+m t r C$ were pregrown 351 anaerobically in varying concentrations of IPTG (ranging from 0 to $1000 \mu \mathrm{M}$ ), leading to differing 352 expression levels of $m t r C$ and resultant EET flux. Cultures were then washed and normalized to $353 \mathrm{OD}_{600}=0.2$ in a reaction mixture containing PAAPEO-ITO, $20 \mathrm{mM}$ lactate, $25 \mu \mathrm{g} / \mathrm{mL}$ kanamycin 
354 for plasmid maintenance, and the corresponding concentration of inducing molecule. As outlined 355 above, spectra were collected through 8 hours and fit with the HEDA model (Figure 5a-d). Fit 356 results are reported in Table S8. As expected, the resultant electron transfer rate during steady357 state was a function of IPTG concentration (Figure 5e), increasing about 5.5-fold from no 358 induction/leaky expression (0 $\mu \mathrm{M}$ IPTG) to full induction (1000 $\mu \mathrm{M}$ IPTG). The consistent linear 359 electron transfer rate across all engineered strains after $1 \mathrm{~h}$ again supports steady-state electron 360 transfer. A wild-type (MR-1), empty vector control grown in 1000 MM IPTG also showed a high 361 electron transfer rate, even higher than the wild-type without a plasmid, which could be attributed 362 to the presence of antibiotic, physiological changes from plasmid maintenance, and/or an 363 anaerobic pregrowth. In this sample, saturation effects were observed at 4 hours, so the rate 364 constant was derived from only the preceding timepoints during steady-state electron transfer 365 (Figures S11-12). It is notable that even the fully induced $m t r C$ complement did not completely 366 recapture the wild-type EET phenotype, exhibiting $\sim 4.5$-fold less activity compared to the empty 367 vector control. This may highlight the importance of other missing EET components in this strain, 368 specifically omcA and $m t r F$, in binding and electron transfer to metal oxide substrates $(53,54)$. 369 Tiered electron flux based on varying gene expression was also quantified as current output, 370 ranging from $0.12 \pm 0.04$ (uninduced) to $0.68 \pm 0.08$ (fully induced) $\mathrm{fA} \cdot \mathrm{cell}^{-1}$ at steady-state (Table

371 2). The rate constants were fit with an activating Hill Function gene expression model as a guide 372 to the eye; as expected, increasing concentrations of IPTG correspond to increasing values of $373 k_{E E T}$ (Figure 5f, Note S4). Together, these results exemplify the quantitative capabilities of our 374 method for measuring electron flux after varying expression levels of a single EET gene. This 375 deepens our understanding of the relationship between mtrC expression and EET output to 376 heterogenous acceptors in planktonic cultures, and may be used to predict electron flux based on $377 m t r C$ expression for a desired application. 


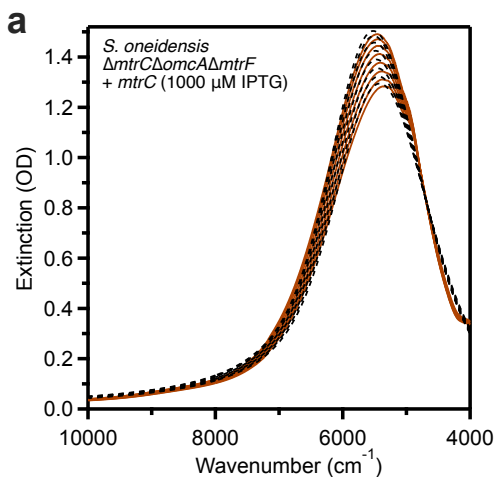

d

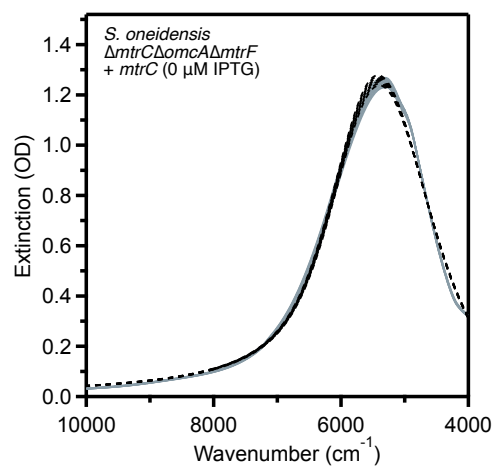

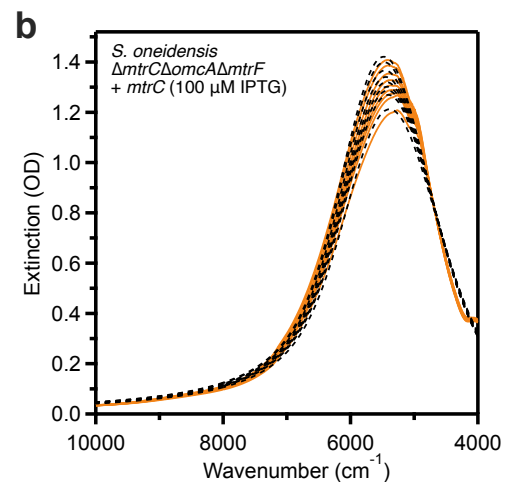

e

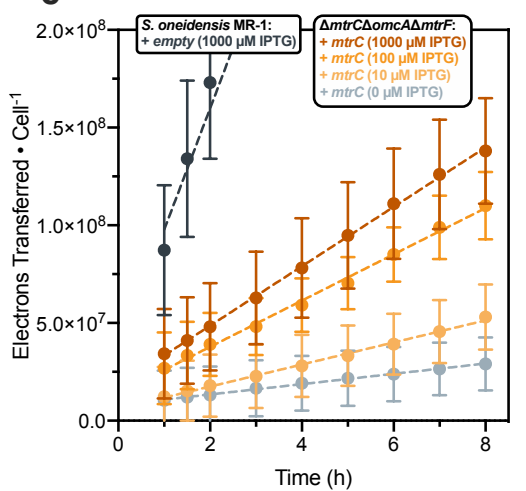

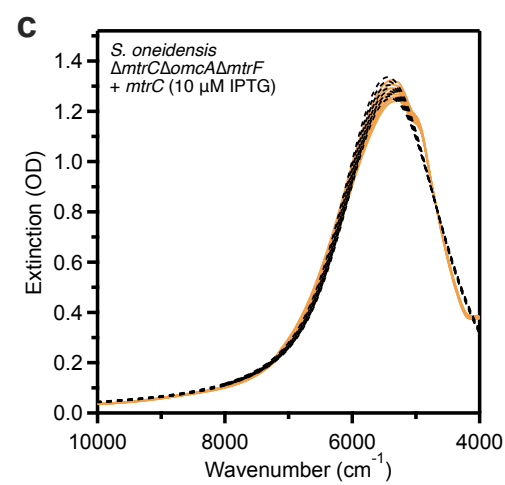

f

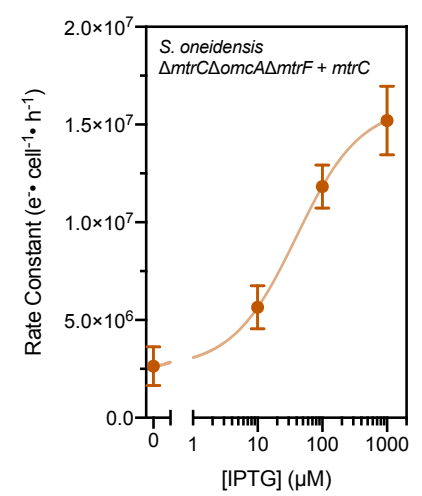

Figure 5. HEDA fitting of PAAPEO-ITO LSPR response during EET from engineered S. oneidensis reveals gradients in population current output as a result of differential gene expression. In situ extinction spectra and HEDA fits for PAAPEO-ITO during EET from (a-d) S. oneidensis $\Delta m$ trC $\Delta$ omcA $\Delta m t r F$ + mtrC with (a) 1000, (b) 100, (c) 10, and (d) $0 \mu \mathrm{M}$ inducing molecule (IPTG). (e) Steady-state EET (1-8 h) where dashed lines represent linear regression of the electrons transferred over time and $k_{E E T}$ is the slope of the regression (Table 2). Data points shown are the mean \pm SD of $n=3$ HEDA model fits of biological replicates. (f) Rate constants exhibit an activating Hill Function-type response to increasing IPTG concentration. Data points shown are the mean \pm SD of $n=3$ independent linear regressions of HEDA model fits of biological replicates.

Table 2. Metrics obtained from HEDA fitting during EET from engineered S. oneidensis MR-1 + empty and $\Delta m t r C \Delta o m c A \Delta m t r F+m t r C$. Data shown are results of the steady-state regime, between 1 and $8 \mathrm{~h}$ for all samples except for MR-1 + empty, which was between 1 and $3 \mathrm{~h}$. Data shown are the mean \pm SD of $n=3$ HEDA model fits of biological replicates. 


\begin{tabular}{|c|c|c|c|}
\hline Sample & $e^{-}$Transferred $\cdot$ Cell-1 & $k_{E E T}\left(e^{-} \cdot\right.$ cell- $\left.^{-1} \cdot h^{-1}\right)$ & Current $\cdot$ Cell- $^{-1}$ (fA) \\
\hline $\begin{array}{c}\text { S. oneidensis MR-1 } \\
+ \text { empty (1000 } \mu \mathrm{M} \text { IPTG) }\end{array}$ & $(3.2 \pm 1.0) \times 10^{8}$ & $(6.2 \pm 1.3) \times 10^{7}$ & $2.76 \pm 0.60$ \\
\hline $\begin{aligned} & \Delta m t r C \Delta o m c A \Delta m t r F \\
+ & m t r C(1000 \mu \mathrm{M} \text { IPTG })\end{aligned}$ & $(1.0 \pm 0.3) \times 10^{8}$ & $(1.5 \pm 0.2) \times 10^{7}$ & $0.68 \pm 0.08$ \\
\hline $\begin{aligned} & \Delta m t r C \Delta o m c A \Delta m t r F \\
+ & m t r C(100 \mu \mathrm{M} \text { IPTG })\end{aligned}$ & $(8.3 \pm 1.9) \times 10^{7}$ & $(1.2 \pm 0.1) \times 10^{7}$ & $0.53 \pm 0.05$ \\
\hline $\begin{array}{l}\Delta m \operatorname{sic} C \Delta o m c A \Delta m t r F \\
+\operatorname{mtrC}(10 \mu \mathrm{M} \text { IPTG })\end{array}$ & $(4.1 \pm 1.3) \times 10^{7}$ & $(5.6 \pm 1.1) \times 10^{6}$ & $0.25 \pm 0.05$ \\
\hline $\begin{array}{l}\Delta m t r C \Delta o m c A \Delta m t r F \\
+m t r C(0 \mu \mathrm{M} I P T G)\end{array}$ & $(1.9 \pm 1.3) \times 10^{7}$ & $(2.6 \pm 1.0) \times 10^{6}$ & $0.12 \pm 0.04$ \\
\hline
\end{tabular}

\section{Conclusions}

399 Together, our results demonstrate that the sensing capabilities of plasmonic ITO nanocrystals in 400 microbial suspensions can expand the microbiologist toolkit for quantifying biological electron 401 transfer. Using our platform, we successfully measured current generation on insoluble substrates 402 from planktonic cultures, enabling differentiation of $S$. oneidensis cells of varying metabolic activity 403 and genotype. In addition, we quantified changes in current generation by cells engineered to 404 express varying levels of a critical EET gene, mtrC. This will enable fundamental studies of 405 biological electron transfer metrics and resolution of EET gene expression dynamics. For 406 example, PAAPEO-ITO biosensors can explore, in real time, how cells change electron flux in 407 response to their environment, including the presence of a new electron acceptor or an inducing 408 molecule. This non-destructive, in situ spectroscopic measurement could also be integrated with 409 high-throughput technologies such as infrared microplate readers to screen genetic mutants or 410 new bacterial strains based on electron transfer rate. In addition, infrared detection of EET 411 enables simultaneous collection of visible wavelength metrics like growth rate, metabolic dyes, 412 and fluorescent protein expression. We also envision PAAPEO-ITO could be used to monitor EET 413 in visibly opaque environments, such as soil, but this will require more experiments to validate.

415 Our recent progress in both surface functionalization and spectroelectrochemical modeling have 416 enabled this demonstration of PAAPEO-ITO as an accurate EET sensing platform; however, 417 further advances in each of these areas will enable even more applications. For example, 418 continuing to develop novel nanocrystal surface chemistry, such as functionalizing with bioactive 419 polymers, could expand our capability to target specific cellular machinery, including surface 420 proteins such as MtrC. In addition, standardizing the optical signatures of ITO nanocrystals with 
known surface potentials could allow both electron tracking and mapping of potential energy landscapes across the cell. Overall, our design highlights concurrent progress in nanocrystal biosensors and synthetic biology for EET, enabling more effective evaluation of critical biological processes.

\section{Materials and Methods}

Chemicals and reagents: Deuterium oxide $\left(\mathrm{D}_{2} \mathrm{O}\right.$, Sigma-Aldrich, 99.9 atom \% $\left.\mathrm{D}\right)$, sodium DL-lactate $\left(\mathrm{NaC}_{3} \mathrm{H}_{5} \mathrm{O}_{3}, \mathrm{TCl}, 60 \%\right.$ in water), sodium fumarate $\left(\mathrm{Na}_{2} \mathrm{C}_{4} \mathrm{H}_{2} \mathrm{O}_{4}\right.$, VWR, 98\%), HEPES buffer solution $\left(\mathrm{C}_{8} \mathrm{H}_{18} \mathrm{~N}_{2} \mathrm{O}_{4} \mathrm{~S}\right.$, VWR, $1 \mathrm{M}$ in water, $\left.\mathrm{pH}=7.3\right)$, potassium phosphate dibasic $\left(\mathrm{K}_{2} \mathrm{HPO}_{4}\right.$, Sigma-Aldrich), potassium phosphate monobasic $\left(\mathrm{KH}_{2} \mathrm{PO}_{4}\right.$, Sigma-Aldrich), sodium chloride ( $\left.\mathrm{NaCl}, \mathrm{VWR}\right)$, ammonium sulfate $\left(\left(\mathrm{NH}_{4}\right)_{2} \mathrm{SO}_{4}\right.$, Fisher Scientific), magnesium (II) sulfate heptahydrate $\left(\mathrm{MgSO}_{4} \cdot 7 \mathrm{H}_{2} \mathrm{O}\right.$, VWR), trace mineral supplement (ATCC), casamino acids (VWR), silicone oil (Alfa Aesar), N,N-dimethylformamide (DMF, Sigma-Aldrich, $\geq 99.8 \%$ ), nitrosonium tetrafluoroborate $\left(\mathrm{NOBF}_{4}\right.$, Sigma-Aldrich, $95 \%$ ), ammonium cerium (IV) nitrate (Sigma-Aldrich, $\geq 98.5 \%$ ), toluene (Fisher Scientific, $\geq 99.5 \%$ ), tin (IV) acetate (Sigma Aldrich), indium (III) acetate (STREM 99.99\%), oleic acid (Sigma Aldrich, technical grade, 90\%), oleyl alcohol Sigma Aldrich, technical grade, $85 \%$ ), hexane (Fisher Scientific, various methylpentanes $4.2 \%$, $\geq 98.5 \%$ ) isopropyl $ß-D-1$-thiogalactopyranoside (IPTG, Teknova), kanamycin sulfate $\left(\mathrm{C}_{18} \mathrm{H}_{38} \mathrm{~N}_{4} \mathrm{O}_{15} \mathrm{~S}\right.$, Growcells), nail polish (Electron Microscopy Sciences), BacLight Live/Dead Stain (Invitrogen) were all used as purchased unless otherwise noted. All media components were autoclaved or sterilized using $0.2 \mu \mathrm{m}$ PES filters.

Nanocrystal synthesis: Tin-doped indium oxide nanocrystals were synthesized using a slow-injection approach pioneered by the Hutchison group $(39,55)$. Indium acetate and tin acetate in a 9:1 molar ratio were dissolved in oleic acid at an overall concentration of $0.5 \mathrm{mmol}$ metal per $\mathrm{mL}$ oleic acid. The solution was heated to $150^{\circ} \mathrm{C}$ and kept at that temperature for at least two hours to convert the metal-acetate complexes into the metal-oleate precursor. Using a syringe pump, the precursor was injected into a hot bath $\left(290^{\circ} \mathrm{C}\right)$ of oleyl alcohol at a rate of $0.3 \mathrm{~mL} / \mathrm{min}$. For this work, $4.5 \mathrm{~mL}$ of precursor was injected. The nanocrystals were collected from the reaction mixture and purified by flocculating with ethanol, centrifuging at $9000 \mathrm{rpm}$, and dispersing in hexane three consecutive times.

Nanocrystal ligand stripping: $\mathrm{NOBF}_{4}$ was used to chemically remove the native organic ligands bound to the nanocrystal surface following a previously established method ${ }^{31}$. Briefly, $60 \mathrm{mg}$ of NOBF 4 were added to a two-phase mixture of DMF $(2 \mathrm{~mL})$ and ITO nanocrystal dispersion in hexane $(\sim 50 \mathrm{mg} / \mathrm{mL}, 2 \mathrm{~mL})$. The mixture was sonicated for $30 \mathrm{~min}$ to promote ligand removal and therefore the phase transfer of nanocrystals from hexane to DMF. After discarding the hexane layer, charge-stabilized ITO nanocrystals dispersed in DMF were purified by performing seven cycles of flocculation with toluene, centrifugation at $7500 \mathrm{rpm}$ for $5 \mathrm{~min}$, and redispersion in DMF.

Nanocrystal polymer wrapping: PAA-mPEO ${ }_{4}$ random copolymer was synthesized $(40,41)$ and ITO nanocrystals were polymer-wrapped with PAA-mPEO following previously established methods (42). Briefly, a PAA-mPEO 4 solution was prepared by dissolving $10 \mathrm{mg}$ of polymer in $700 \mu \mathrm{L}$ of DMF. Then, 300 $\mu \mathrm{L}$ of ITO nanocrystal dispersed in DMF $(\sim 90 \mathrm{mg} / \mathrm{mL})$ were added dropwise to the PAA-mPEO 4 solution under gentle stirring. This nanocrystal-polymer mixture was stirred overnight and subsequently added dropwise into $19 \mathrm{~mL}$ of Milli- $Q$ water while stirring. The aqueous mixture was stirred at $600 \mathrm{rpm}$ for 48 hours. Polymer-wrapped ITO nanocrystals were recovered and purified by performing three cycles of spin dialysis (centrifugation at $4000 \mathrm{rpm}$ for $10 \mathrm{~min}$ per cycle) using $50 \mathrm{kDa}$ Millipore Amicon Ultra centrifugal tubes. The final dispersion in Milli-Q water was filtered through a $0.45 \mu \mathrm{m}$ PVDF syringe filter (Acrodisc, Pall).

Oxidation of polymer-wrapped nanocrystals: Polymer-wrapped ITO nanocrystals dispersed in Milli- $Q$ water were chemically oxidized following a previously established method (36). Briefly, $120 \mu \mathrm{L}$ of polymerwrapped ITO $(\sim 7 \mathrm{mg} / \mathrm{mL})$ were mixed with $25 \mu \mathrm{L}$ of an aqueous solution of ammonium cerium nitrate (25 $\mathrm{mM})$. Instead of scaling up the volume of the reaction, the reagents were combined in multiple separate 
vials to achieve the desired final mass of oxidized and polymer-wrapped nanocrystals. The mixture was left to stand without stirring for $15 \mathrm{~min}$ and then purified by performing three cycles of spin dialysis (centrifugation at $4000 \mathrm{rpm}$ for $10 \mathrm{~min}$ per cycle) using $50 \mathrm{kDa}$ Millipore Amicon Ultra centrifugal tubes. The resulting oxidized and polymer-wrapped ITO nanocrystals were then dispersed in deuterated SBM + CAAs (Table S1) by performing a solvent exchange through three additional spin dialysis cycles with excess deuterated buffer instead of Milli-Q water (final concentration: $1.5 \mathrm{mg} / \mathrm{mL}$ ).

Nanocrystal Characterization: a. Electron microscopy. ITO nanocrystals were imaged in bright-field scanning transmission electron microscopy mode at a $30 \mathrm{kV}$ accelerating voltage on a Hitachi S5500 SEM/STEM instrument. The sample was drop-cast on Type-A ultrathin carbon copper TEM grids (Ted Pella, 01822, 400 mesh) from dilute ITO dispersions in hexane. b. Small-angle X-ray scattering (SAXS). The size of as-synthesized ITO nanocrystals was determined by SAXS. A dilute dispersion $(\sim 1 \mathrm{mg} / \mathrm{mL})$, enclosed in a sealed glass capillary (Charles-Supper Company, Boron Rich, $1.5 \mathrm{~mm}$ diameter, $0.01 \mathrm{~mm}$ wall thickness), was measured in transmission configuration at a sample-detector distance of $1 \mathrm{~m}$ on a SAXSLAB Ganesha instrument using $\mathrm{Cu} \mathrm{K \alpha}$ radiation. Details of SAXS data analysis are included in the Supplementary Information. c. Dynamic light scattering. The hydrodynamic diameters of ligand-stripped, polymer-wrapped, and oxidized ITO nanocrystals were measured on a Malvern Zetasizer Nano ZS. Dilute ITO nanocrystals dispersions $(\sim 1 \mathrm{mg} / \mathrm{mL}$ ) were enclosed in disposable plastic micro cuvettes (ZEN0040, Malvern). d. Thermogravimetric analysis. PAA-mPEO 4 adsorption on the ITO nanocrystal surface was quantified using a Mettler Toledo TGA 2. In a typical experiment, $100 \mu \mathrm{L}$ of polymer-wrapped ITO nanocrystals dispersed in Milli-Q water $(\sim 10 \mathrm{mg} / \mathrm{mL})$ was added to a disposable aluminum crucible and dried under vacuum at room temperature for 24 hours. The sample was measured in dry synthetic air from $30^{\circ} \mathrm{C}$ to $600{ }^{\circ} \mathrm{C}$ at a $5^{\circ} \mathrm{C} / \mathrm{min}$ ramp rate. e. Fourier transform infrared spectroscopy. Ligand-capped, ligand-stripped, and polymer-wrapped ITO nanocrystals were measured in transmission geometry with a Bruker Vertex 70 spectrometer. Samples were drop-cast from dilute nanocrystal dispersion $(\sim 1 \mathrm{mg} / \mathrm{mL})$ on undoped, double side polished silicon substrates (Virginia Semiconductor Inc.) f. UV/Vis/NIR Spectroscopy. The cuvettes were placed in an Agilent Cary series ultraviolet-visible-near infrared spectrophotometer in transmission mode for spectra collection. Spectra were collected from 3032-10000 $\mathrm{cm}^{-1}$ for a collection time of $\sim 1$ min per spectrum. g. Inductively coupled plasma-optical emission spectroscopy (ICP-OES). To quantify the volume fraction of nanocrystals in dispersion, as well as the doping percentage of tin atoms in the indium oxide lattice, we took a known volume of the dispersed nanocrystals, dried them into a pellet and dissolved them in aqua regia for two days. We then diluted the dissolved ions to $2 \% \mathrm{v} / \mathrm{v}$ acid and loaded the dilute acid into the Varian 720 -ES ICP-OES to quantify the tin and indium concentrations. We calibrated the instrument for tin and indium using standards purchased from Sigma Aldrich (TraceCERT® 1000mg In/L nitric acid and 1000mg Sn/L nitric acid).

Bacterial strains and culture: Bacterial strains and plasmids are listed in Table S3. Cultures were prepared as follows: bacterial stocks stored in $22.5 \%$ glycerol at $-80^{\circ} \mathrm{C}$ were streaked onto LB agar plates (for wild-type and knockout strains) or LB agar with $25 \mu \mathrm{g} / \mathrm{mL}$ kanamycin (for plasmid-harboring strains) and grown overnight at $30^{\circ} \mathrm{C}$ for Shewanella, $37^{\circ} \mathrm{C}$ for E. coli. Single colonies were isolated and inoculated into Shewanella Basal Medium (SBM) supplemented with $100 \mathrm{mM}$ HEPES, $0.05 \%$ trace mineral supplement (Table S2), $0.05 \%$ casamino acids, and $20 \mathrm{mM}$ sodium lactate $(2.85 \mu \mathrm{L}$ of $60 \% \mathrm{w} / \mathrm{w}$ sodium lactate per $1 \mathrm{~mL}$ culture) as the electron donor. Aerobic cultures were pregrown in $15 \mathrm{~mL}$ culture tubes at $30{ }^{\circ} \mathrm{C}$ and $250 \mathrm{rpm}$ shaking. Anaerobic, plasmid-harboring cultures were pregrown using the same procedure outlined above, but in degassed growth medium in a humidified anaerobic chamber, supplemented with $40 \mathrm{mM}$ sodium fumarate $(40 \mu \mathrm{L} / \mathrm{mL}$ of a $1 \mathrm{M}$ stock) as the electron acceptor, $25 \mu \mathrm{g} / \mathrm{mL}$ kanamycin, and varying concentrations of IPTG. Descriptions of the mtrC circuit and pCD24r1 plasmid, as well as the empty vector circuit and the pCD8 plasmid, have been published previously (12). Cultures were washed $3 x$ after pregrowth using SBM supplemented with $0.5 \%$ casamino acids (degassed for anaerobic cultures). Before the final centrifugation, OD 600 was measured using a NanoDrop 2000C spectrophotometer and the culture volume normalized such that the final OD600 in the reaction with PAAPEO-ITO would be 0.2 .

Viability assessments: All microscopy was performed using a Nikon Ti2 Eclipse inverted epifluorescence microscope. Cells assessed for viability by microscopy were incubated with PAAPEO-ITO for $2 \mathrm{~h}$ at $30^{\circ} \mathrm{C}$. 529 The cells were centrifuged, and the nanocrystals were washed away using $0.85 \% \mathrm{NaCl}$ solution. The cell 
suspensions were then incubated in the dark in the BacLight Live/Dead stain mix $(1.5 \mu \mathrm{L} / \mathrm{mL}$ Syto9, 2.5 $\mu \mathrm{L} / \mathrm{mL}$ propidium iodide in $0.85 \% \mathrm{NaCl}$ solution) for 20 minutes. Stained cells were then washed $3 \mathrm{x}$ in $0.85 \%$ $\mathrm{NaCl}$ solution to remove unbound dye. Cell suspensions were loaded onto glass microscope slides and a no. 1 coverslip placed on top. Nail polish was used to seal the coverslip to the glass slide to prevent evaporative losses. Fluorescence for each stain (green for Syto9, red for propidium iodide) was measured using GFP and Texas Red excitation/emission filter cubes on a Nikon Ti2 Eclipse, as outlined previously (7). Live and dead populations were quantified using thresholding and masking in Fiji 1.0. For colony counting, cell samples before and after incubation with PAAPEO-ITO were diluted by factors of $10^{5}-10^{7}$ in LB medium, and $100 \mu \mathrm{L}$ of these dilutions was plated onto LB-agar. The viable population (CFU/mL) for each sample was quantified by averaging $\frac{\mathrm{CFU}}{\mathrm{mL}}=\frac{\# \text { of colonies } \bullet \text { dilution }}{\left(100 \mathrm{\mu L} / 1000 \mu \mathrm{L} \cdot \mathrm{mL}^{-1}\right)}$ for each replicate.

\section{Electron transfer experiments and spectroscopy measurements:}

Bacteria and PAAPEO-ITO nanocrystals were mixed in a disposable centrifuge tube and immediately transferred to a Spectrocell near-IR quartz cuvette and the cuvette was sealed for the duration of the experiment. The cuvette was loaded into an Agilent Cary UV/vis/NIR spectrophotometer for spectra collection. On average, the first spectrum was taken $\sim 3$ min after mixing. Thereafter, a spectrum was collected every 10 minutes for the first hour and then every hour until 8 hours. Three cuvettes were used to carry out triplicate experiments simultaneously, two with pathlengths of $1 \mathrm{~mm}$ and the third with pathlength $0.5 \mathrm{~mm}$. All spectra were backgrounded to a cuvette filled solely with deuterated SBM buffer, without PAAPEO-ITO or bacteria.

Author Contributions: A.J.G., S.L.G., C.A.S.C., D.J.M., and B.K.K. conceived the project; A.J.G., S.L.G., C.A.S.C., Y.W., and A.M.G. performed experiments; S.L.G., C.A.S.C., and Y.W. performed nanocrystal synthesis and characterization; A.J.G. designed biological experiments and performed microbial culture; A.J.G., S.L.G., C.A.S.C., D.J.M., and B.K.K. analyzed the results; A.J.G., S.L.G., C.A.S.C., D.J.M., and B.K.K. wrote the manuscript with input from all authors.

Acknowledgements: The authors declare that they have no competing interests. All data needed to evaluate the conclusions in the paper are present in the paper and/or the Supplementary Materials. $S$. oneidensis $\Delta m t r C \Delta o m c A \Delta m t r F$ was a generous gift from Prof. Jeffrey Gralnick (U. Minnesota), and $E$. coli MG1655 and S. oneidensis S2933 were generous gifts from Prof. Lydia Contreras (UT Austin). Christopher Dundas is thanked for his contribution of the engineered S. oneidensis strain. A.J.G. and S.L.G. were supported through National Science Foundation Graduate Research Fellowships (Program Award No. DGE-1610403). Portions of this work, including polymer synthesis and characterization by Swagat Sahu and Brett A. Helms, were carried out as a user project at the Molecular Foundry, which is supported by the Office of Science, Office of Basic Energy Sciences of the U.S. Department of Energy under contract no. DE-AC02-05CH11231. This research was primarily supported by the National Science Foundation through the Center for Dynamics and Control of Materials: an NSF Materials Research Science and Engineering Center under DMR-1720595. Additional research support was provided by the Welch Foundation (Grants F-1929 and F-1848), the National Institute of General Medical Sciences of the National Institutes of Health under Award Number R35GM133640 (B.K.K.), and an NSF CAREER Award (1944334, B.K.K.). The authors acknowledge use of shared research facilities supported in part by the Texas Materials Institute including the SAXS instrument acquired under an NSF MRI grant (CBET-1624659), the Center for Dynamics and Control of Materials: an NSF MRSEC (DMR-1720595), and the NSF National Nanotechnology Coordinated Infrastructure (ECCS-1542159). We gratefully acknowledge the use of facilities within the core microscopy lab of the Institute for Cellular and Molecular Biology, University of Texas at Austin.

Data Deposition: Experimental data supporting the findings in this study are publicly available through the Texas Data Repository (doi: https://doi.org/10.18738/T8/OU8RNO).

\section{ORCID:}

Austin J. Graham: 0000-0001-8924-181X

Stephen L. Gibbs: 0000-0003-2533-0957

Camila A. Saez Cabezas: 0000-0002-8734-0096 
Allison M. Green: 0000-0002-7734-4202

Delia J. Milliron: 0000-0002-8737-451X

Benjamin K. Keitz: 0000-0003-3314-0053

\section{References}

1. L. Shi, H. Dong, G. Reguera, H. Beyenal, A. Lu, J. Liu, H.-Q. Yu, J. K. Fredrickson, Extracellular electron transfer mechanisms between microorganisms and minerals. Nat. Rev. Microbiol. 14, 651-662 (2016).

2. J. M. Flynn, D. E. Ross, K. A. Hunt, D. R. Bond, J. A. Gralnick, Enabling Unbalanced Fermentations by Using Engineered Electrode-Interfaced Bacteria. mBio 1, e00190-10-e00190-17 (2010).

3. D. R. Bond, "Electrodes as electron acceptors, and the bacteria who love them" in Geomicrobiology: Molecular and Environmental Perspective, L. Barton, M. Mandl, A. Loy, Eds. (Springer, 2010), pp. 385399.

4. D. E. Ross, J. M. Flynn, D. B. Baron, J. A. Gralnick, D. R. Bond, Towards Electrosynthesis in Shewanella: Energetics of Reversing the Mtr Pathway for Reductive Metabolism. PLoS ONE 6, e16649-9 (2011).

5. Y. Gao, M. Mohammadifar, S. Choi, From Microbial Fuel Cells to Biobatteries: Moving toward OnDemand Micropower Generation for Small-Scale Single-Use Applications. Adv. Mater. Technologies 4, 1900079 (2019).

6. X. Fang, S. Kalathil, G. Divitini, Q. Wang, E. Reisner, A three-dimensional hybrid electrode with electroactive microbes for efficient electrogenesis and chemical synthesis. Proc. Natl. Acad. Sci. U.S.A. 9, 5074-5080 (2020).

7. G. Fan, C. M. Dundas, A. J. Graham, N. A. Lynd, B. K. Keitz, Shewanella oneidensis as a living electrode for controlled radical polymerization. Proc. Natl. Acad. Sci. U.S.A. 4, 4559-4564 (2018).

8. C. M. Dundas, A. J. Graham, D. K. Romanovicz, B. K. Keitz, Extracellular Electron Transfer by Shewanella oneidensis Controls Palladium Nanoparticle Phenotype. ACS Synth. Biol. 7, 2726-2736 (2018).

9. N. Schuergers, C. Werlang, C. M. Ajo-Franklin, A. A. Boghossian, A synthetic biology approach to engineering living photovoltaics. Energ. Environ. Sci. 10, 1102-1115 (2017).

10. B. Tian, S. Xu, J. A. Rogers, S. Cestellos-Blanco, P. Yang, J. L. Carvalho-de-Souza, F. Bezanilla, J. Liu, Z. Bao, M. Hjort, Y. Cao, N. Melosh, G. Lanzani, F. Benfenati, G. Galli, F. Gygi, R. Kautz, A. A. Gorodetsky, S. S. Kim, T. K. Lu, P. Anikeeva, M. Cifra, O. Krivosudský, D. Havelka, Y. Jiang, Roadmap on semiconductor-cell biointerfaces. Phys Biol 15, 031002 (2018).

11. D. Coursolle, J. A. Gralnick, Modularity of the Mtr respiratory pathway of Shewanella oneidensis strain MR-1. Mol. Microbiol. 55, 995-1008 (2010).

12. C. M. Dundas, D. J. F. Walker, B. K. Keitz, Tuning Extracellular Electron Transfer by Shewanella oneidensis Using Transcriptional Logic Gates. ACS Synth. Biol. 9, 2301-2315 (2020). 
13. J. T. Atkinson, I. J. Campbell, E. E. Thomas, S. C. Bonitatibus, S. J. Elliott, G. N. Bennett, J. J. Silberg, Metalloprotein switches that display chemical- dependent electron transfer in cells. Nat. Chem. Biol. 15, 189-195 (2018).

14. D. Coursolle, D. B. Baron, D. R. Bond, J. A. Gralnick, The Mtr Respiratory Pathway Is Essential for Reducing Flavins and Electrodes in Shewanella oneidensis. J. of Bacteriol. 192, 467-474 (2009).

15. A. R. Rowe, P. Rajeev, A. Jain, S. Pirbadian, A. Okamoto, J. A. Gralnick, M. Y. El-Naggar, K. H. Nealson, Tracking Electron Uptake from a Cathode into Shewanella Cells: Implications for Energy Acquisition from Solid-Substrate Electron Donors. mBio 9, e02203-17-19 (2018).

16. Q. Wang, A.-A. D. Jones, J. A. Gralnick, L. Lin, C. R. Buie, Microfluidic dielectrophoresis illuminates the relationship between microbial cell envelope polarizability and electrochemical activity. Sci. Adv. 5, eaat5664 (2019).

17. H. Liu, G. J. Newton, R. Nakamura, K. Hashimoto, S. Nakanishi, Electrochemical Characterization of a Single Electricity-Producing Bacterial Cell of Shewanella by Using Optical Tweezers. Angew. Chem. Int. 49, 6596-6599 (2010).

18. B. J. Gross, M. Y. El-Naggar, A combined electrochemical and optical trapping platform for measuring single cell respiration rates at electrode interfaces. Rev. Sci. Instrum. 86, 064301-9 (2015).

19. S. Pirbadian, M. S. Chavez, M. Y. El-Naggar, Spatiotemporal mapping of bacterial membrane potential responses to extracellular electron transfer. Proc. Natl. Acad. Sci. U.S.A. 117, 20171-20179 (2020).

20. G. Méhes, A. Roy, X. Strakosas, M. Berggren, E. Stavrinidou, D. T. Simon, Organic Microbial Electrochemical Transistor Monitoring Extracellular Electron Transfer. Adv. Sci. 7, 2000641 (2020).

21. H. H. Hau, J. A. Gralnick, Ecology and Biotechnology of the Genus Shewanella. Ann. Rev. of Microbiol. 61, 237-258 (2007).

22. E. D. Brutinel, J. A. Gralnick, Shuttling happens: soluble flavin mediators of extracellular electron transfer in Shewanella. Appl. Microbiol. Biot. 93, 41-48 (2012).

23. F. Zhang, J.-H. Wu, H.-Q. Yu, Probing Microbial Extracellular Respiration Ability Using Riboflavin. Anal. Chem. 15, 10606-10612 (2020).

24. A. M. Schimpf, C. E. Gunthardt, J. D. Rinehart, J. M. Mayer, D. R. Gamelin, Controlling Carrier Densities in Photochemically Reduced Colloidal ZnO Nanocrystals: Size Dependence and Role of the Hole Quencher. J. Am. Chem. Soc. 135, 16569-16577 (2013).

25. A. M. Schimpf, S. D. Lounis, E. L. Runnerstrom, D. J. Milliron, D. R. Gamelin, Redox Chemistries and Plasmon Energies of Photodoped $\operatorname{In}_{2} \mathrm{O}_{3}$ and Sn-Doped $\operatorname{In}_{2} \mathrm{O}_{3}$ (ITO) Nanocrystals. J. Am. Chem. Soc. 137, 518-524 (2015).

26. A. Agrawal, I. Kriegel, E. L. Runnerstrom, F. Scotognella, A. Llordes, D. J. Milliron, Rationalizing the Impact of Surface Depletion on Electrochemical Modulation of Plasmon Resonance Absorption in Metal Oxide Nanocrystals. ACS Photon. 5, 2044-2050 (2018).

27. A. Agrawal, S. H. Cho, O. Zandi, S. Ghosh, R. W. Johns, D. J. Milliron, Localized Surface Plasmon Resonance in Semiconductor Nanocrystals. Chem. Rev. 118, 3121-3207 (2018). 
663

664

665

666

667

668

669

670

671

672

673

674

675

676

677

678

679

680

681

682

683

684

685

686

687

688

689

690

691

692

693

694

695

696

697

698

699

28. R. S. Lakes, Negative-Poisson's-Ratio Materials: Auxetic Solids. Ann. Rev. Mater. Res. 47, 63-81 (2017).

29. A. Dong, X. Ye, J. Chen, Y. Kang, T. Gordon, J. M. Kikkawa, C. B. Murray, A Generalized LigandExchange Strategy Enabling Sequential Surface Functionalization of Colloidal Nanocrystals. J. Am.

Chem. Soc. 133, 998-1006 (2011).

30. J. D. Roo, F. van den Broeck, K. De Keukeleere, J. C. Martins, I. Van Driessche, Z. Hens, Unravelling the Surface Chemistry of Metal Oxide Nanocrystals, the Role of Acids and Bases. J. Am. Chem. Soc. 136, 9650-9657 (2014).

31. R. M. Dragoman, M. Grogg, M. I. Bodnarchuk, P. Tiefenboeck, D. Hilvert, D. N. Dirin, M. V. Kovalenko, Surface-Engineered Cationic Nanocrystals Stable in Biological Buffers and High lonic Strength Solutions. Chem. Mater. 29, 9416-9428 (2017).

32. P. Chandrangsu, C. Rensing, J. D. Helmann, Metal homeostasis and resistance in bacteria. Nat. Rev. Microbiol. 15, 338-350 (2017).

33. H. Richter, M. Lanthier, K. P. Nevin, D. R. Lovley, Lack of Electricity Production by Pelobacter carbinolicus Indicates that the Capacity for Fe(III) Oxide Reduction Does Not Necessarily Confer Electron Transfer Ability to Fuel Cell Anodes. Appl. Environ. Microb. 73, 5347-5353 (2007).

34. S.-J. Yuan, H. He, G.-P. Sheng, J.-J. Chen, Z.-H. Tong, Y.-Y. Cheng, W.-W. Li, Z.-Q. Lin, F. Zhang, H.-Q. Yu, A Photometric High-Throughput Method for Identification of Electrochemically Active Bacteria Using a $\mathrm{WO}_{3}$ Nanocluster Probe. Sci. Rep. 3, 1315 (2013).

35. L. Su, T. Fukushima, A. Prior, M. Baruch, T. J. Zajdel, C. M. Ajo-Franklin, Modifying Cytochrome $c$ Maturation Can Increase the Bioelectronic Performance of Engineered Escherichia coli. ACS Synth. Biol. 9, 115-124 (2019).

36. R. J. Mendelsberg, P. M. McBride, J. T. Duong, M. J. Bailey, A. Llordes, D. J. Milliron, B. A. Helms, Dispersible Plasmonic Doped Metal Oxide Nanocrystal Sensors that Optically Track Redox Reactions in Aqueous Media with Single-Electron Sensitivity. Adv. Opt. Mater. 3, 1293-1300 (2015).

37. Y. Cao, M. Song, F. Li, C. Li, X. Lin, Y. Chen, Y. Chen, J. Xu, Q. Ding, H. A. Song, Synthetic Plasmid Toolkit for Shewanella oneidensis MR-1. Front. Microbiol. 10, 410 (2019).

38. S. L. Gibbs, C. M. Staller, A. Agrawal, R. W. Johns, C. A. Saez Cabezas, D. J. Milliron, Intrinsic Optical and Electronic Properties from Quantitative Analysis of Plasmonic Semiconductor Nanocrystal Ensemble Optical Extinction 124, 24351-24360 (2020).

39. A. W. Jansons, J. E. Hutchison, Continuous Growth of Metal Oxide Nanocrystals: Enhanced Control of Nanocrystal Size and Radial Dopant Distribution. ACS Nano 10, 6942-6951 (2016).

40. J. F. Duong, M. J. Bailey, T. E. Pick, P. M. McBride, E. L. Rosen, R. Buonsanti, D. J. Milliron, B. A. Helms, Efficient polymer passivation of ligand-stripped nanocrystal surfaces. J. Polym. Sci. A Polym. Chem. 50, 3719-3727 (2012).

41. M. J. Bailey, R. van der Weegen, P. J. Klemm, S. L. Baker, B. A. Helms, Stealth Rare Earth Oxide Nanodiscs for Magnetic Resonance Imaging. Adv. Healthcare Mater. 1, 437-442 (2012). 
42. A. Maho, C. A. Saez Cabezas, K. A. Meyertons, L. C. Reimnitz, S. Sahu, B. A. Helms, D. J. Milliron, Aqueous Processing and Spray Deposition of Polymer-Wrapped Tin-Doped Indium Oxide Nanocrystals as Electrochromic Thin Films. Chem. Mater. 32, 8401-8411 (2020).

43. K. F. Palmer, D. Williams, Optical properties of water in the near infrared. J. Opt. Soc. Am. 64, 1107

704 (1974).

705

706

707

708

709

44. Y. Zhang, N. V. Hudson-Smith, S. D. Frand, M. S. Cahill, L. S. Davis, Z. V. Feng, C. L. Haynes, R. J. Hamers, Influence of spatial distribution of cationic functional groups at nanoparticle surfaces on bacterial viability and membrane interactions. J. Am. Chem. Soc. 142, 10814-10823 (2020).

45. G. Fan, A. J. Graham, J. Kolli, N. A. Lynd, B. K. Keitz, Aerobic radical polymerization mediated by microbial metabolism. Nat. Chem. 12, 638-646 (2020).

46. A. J. Graham, C. M. Dundas, A. Hillsley, D. S. Kasprak, A. M. Rosales, B. K. Keitz, Genetic Control of Radical Cross-linking in a Semisynthetic Hydrogel. ACS Biomater. Sci. Eng. 6, 1375-1386 (2020).

47. K. A. Hunt, J. M. Flynn, B. Naranjo, I. D. Shikhare, J. A. Gralnick, Substrate-Level Phosphorylation is the Primary Source of Energy Conservation during Anaerobic Respiration of Shewanella oneidensis Strain MR-1. J. of Bacteriol. 192, 3345-3351 (2010).

48. L. Binnenkade, L. Teichmann, K. M. Thormann, Iron Triggers $\lambda$ So Prophage Induction and Release of Extracellular DNA in Shewanella oneidensis MR-1 Biofilms. Appl. Environ. Microb. 80, 5304-5316 (2014).

49. J. S. McLean, G. Wagner, Y. A. Gorby, M. Wainstein, J. McQuaid, S. I. Ishii, O. Bretschger, H. Beyenal, K. H. Nealson, Quantification of Electron Transfer Rates to a Solid Phase Electron Acceptor through the Stages of Biofilm Formation From Single Cells to Multicellular Communities. Environ. Sci. Technol. 44, 2721-2727 (2010).

50. S. Bose, M. F. Hochella Jr., Y. A. Gorby, D. W. Kennedy, D. E. McCready, A. S. Madden, B. H. Lower, Bioreduction of hematite nanoparticles by the dissimilatory iron reducing bacterium Shewanella oneidensis MR-1. Geochim. Cosmochim. Acta 73, 962-976 (2009).

51. S. Xu, Y. Jangir, M. Y. El-Naggar, Disentangling the roles of free and cytochrome-bound flavins in extracellular electron transport from Shewanella oneidensis MR-1. Electrochim. Acta 198, 49-55 (2016).

52. A. Okamoto, Y. Tokunou, S. Kalathil, K. Hashimoto, Proton Transport in the Outer-Membrane Flavocytochrome Complex Limits the Rate of Extracellular Electron Transport. Angew. Chem. 129, 92109214 (2017).

53. T. Fukushima, S. Gupta, B. Rad, J. A. Cornejo, C. J. Petzold, L. J. G. Chan, R. A. Mizrahi, C. Y. Ralston, C. M. Ajo-Franklin, The Molecular Basis for Binding of an Electron Transfer Protein to a Metal Oxide Surface. J. Am. Chem. Soc. 139, 12647-12654 (2017).

54. X. Jing, Y. Wu, L. Shi, C. L. Peacock, N. M. Ashry, C. Gao, Q. Huang, P. Cai, Outer membrane c-type cytochromes OmcA and MtrC play distinct roles in enhancing the attachment of Shewanella oneidensis MR-1 cells to geothite. Appl. Environ. Microbiol. 86, e01941-20 (2020).

55. D. Ito, S. Yokoyama, T. Zaikova, K. Masuko, J. E. Hutchison, Synthesis of Ligand-Stabilized Metal Oxide Nanocrystals and Epitaxial Core/Shell Nanocrystals via a Lower-Temperature Esterification Process. ACS Nano 8, 64-75 (2014). 
a

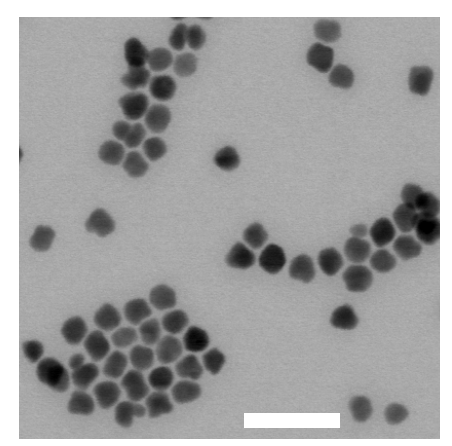

b

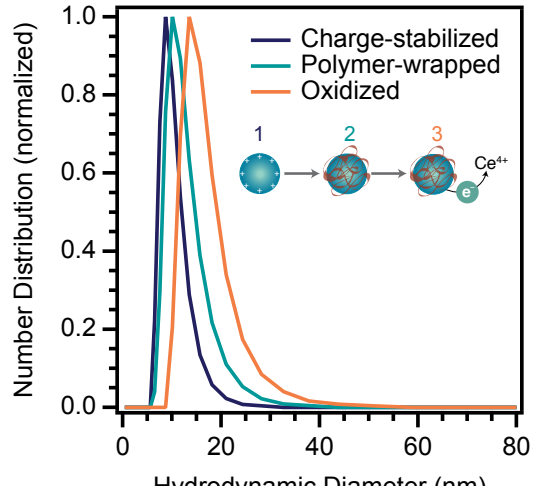

C

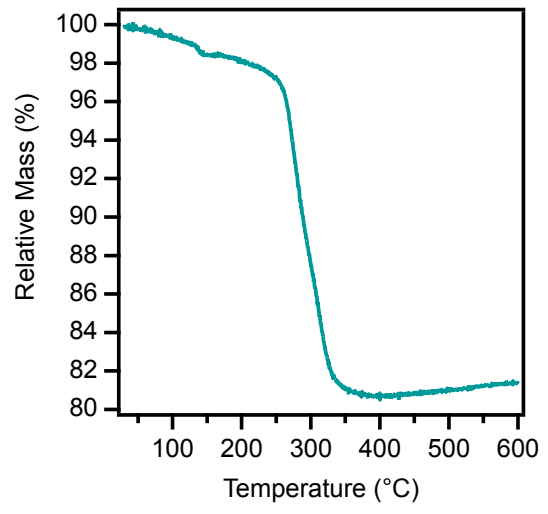


a

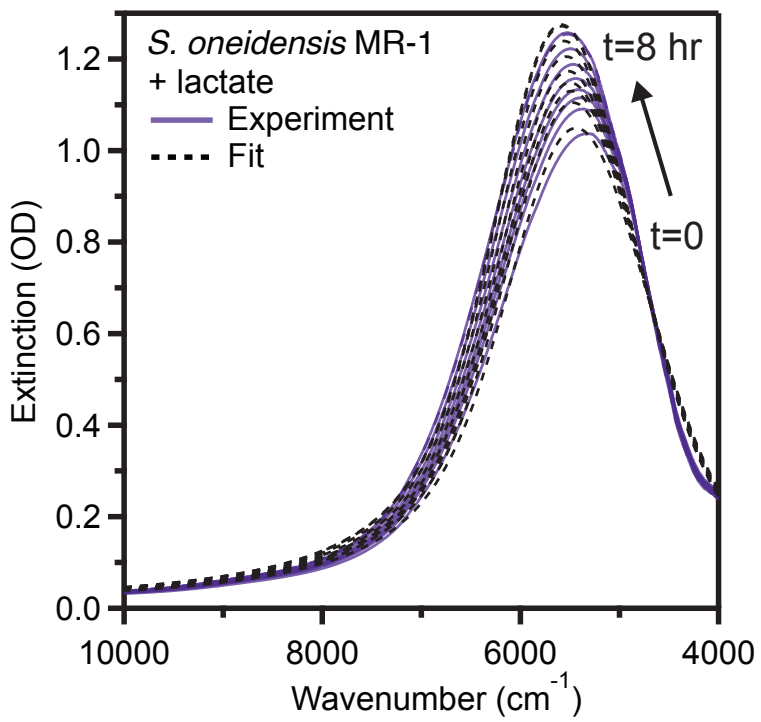

b

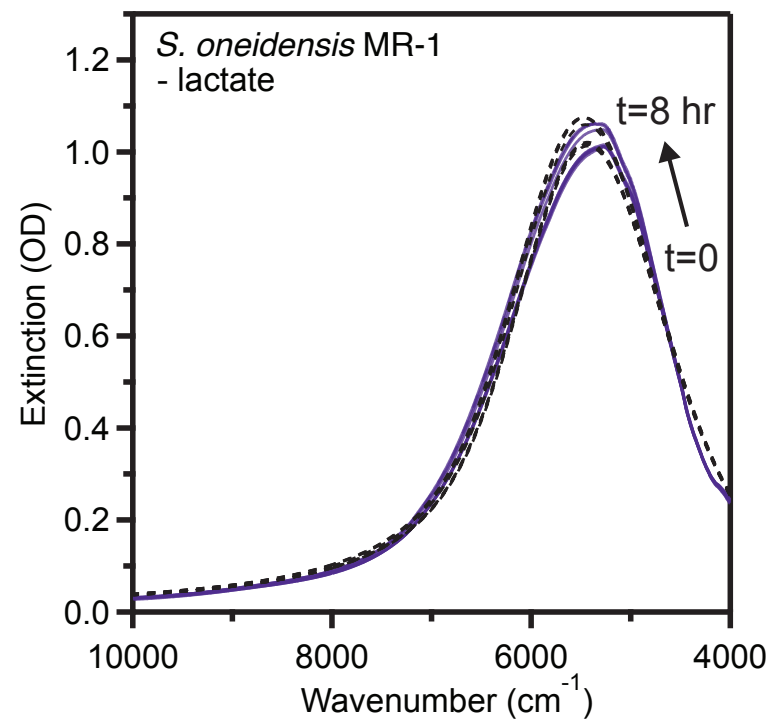

C

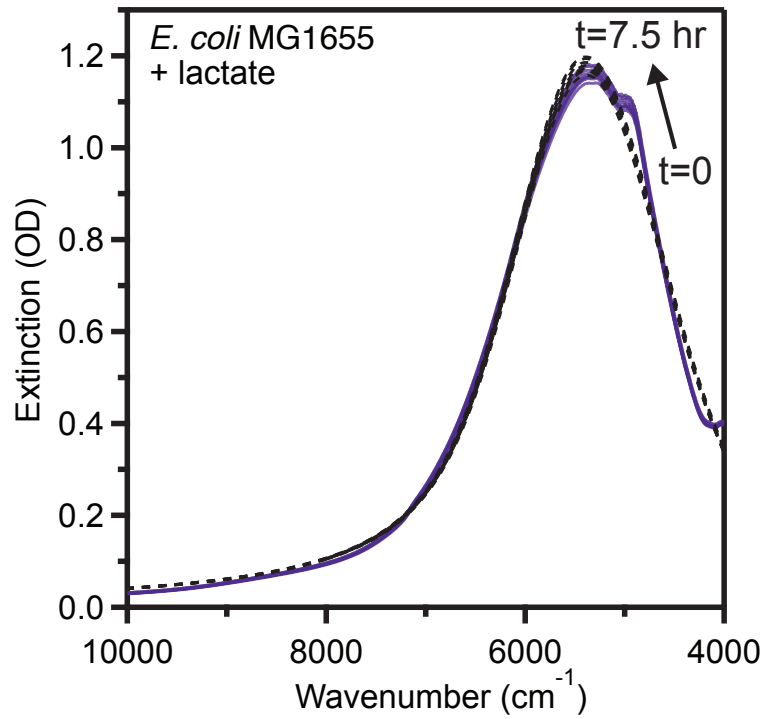


a

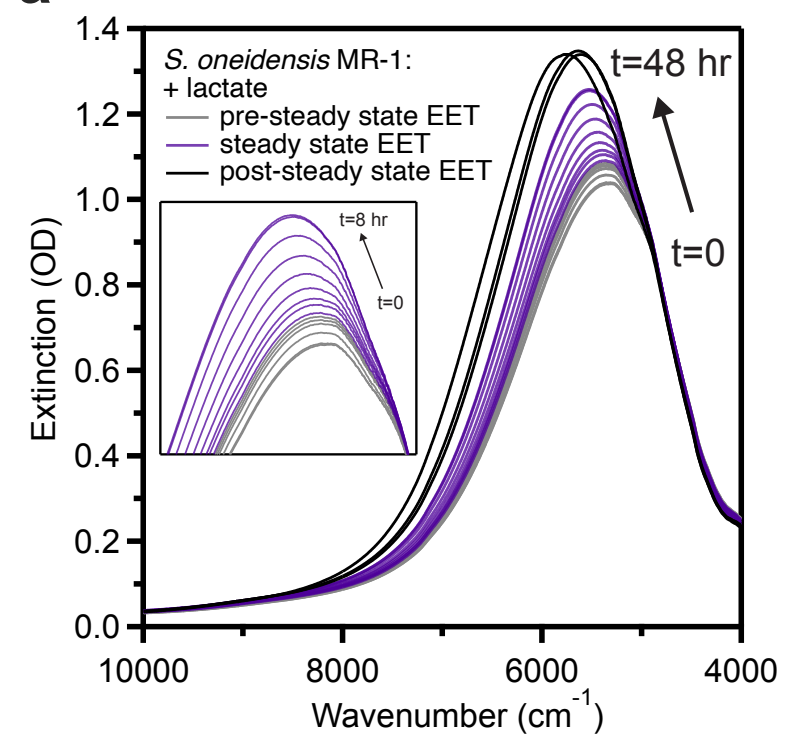

b

\section{S. oneidensis} MR-1:

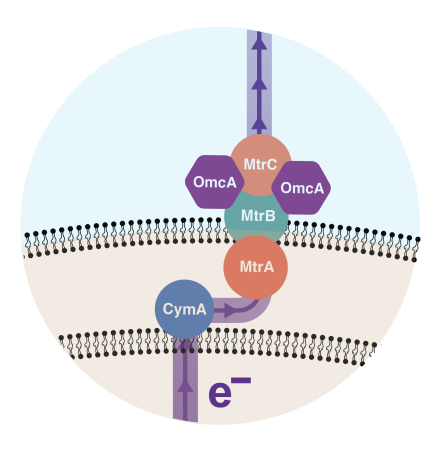

S. oneidensis

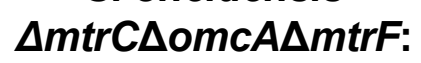

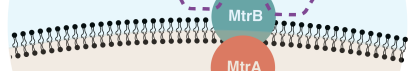

$\mathrm{e}^{-}$
C

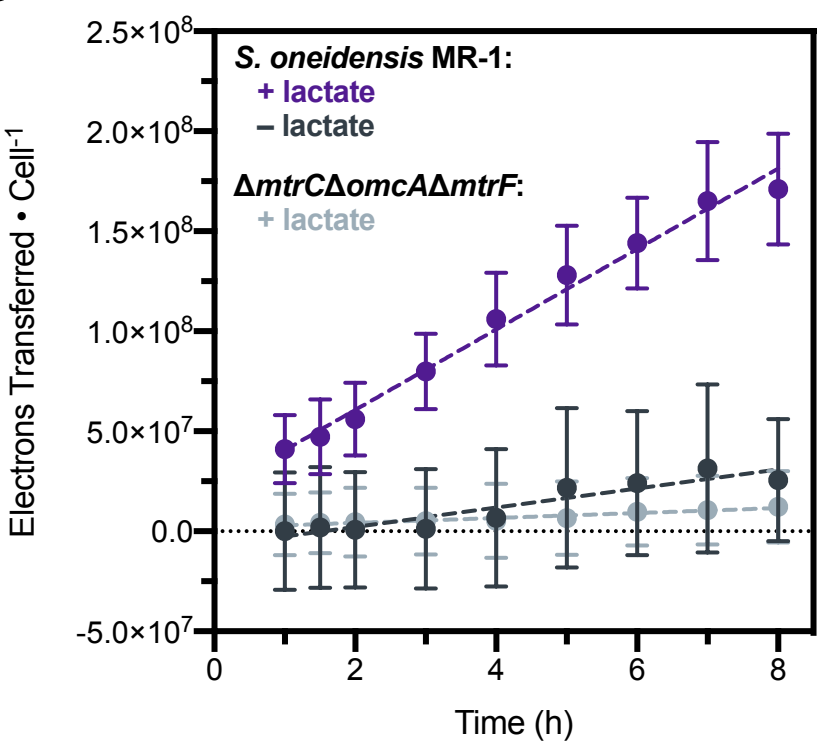


a

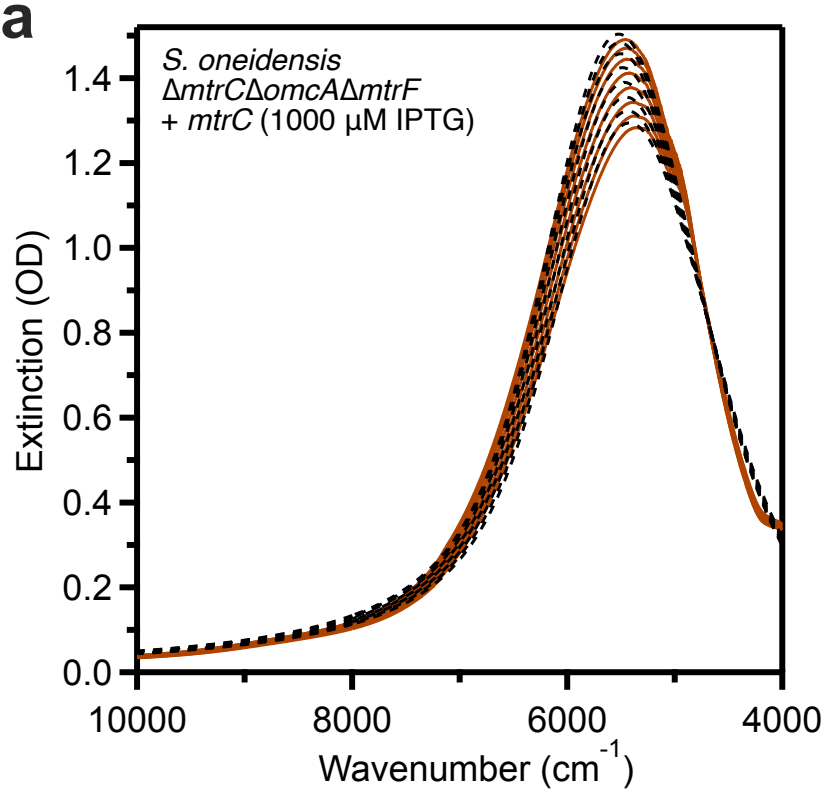

d

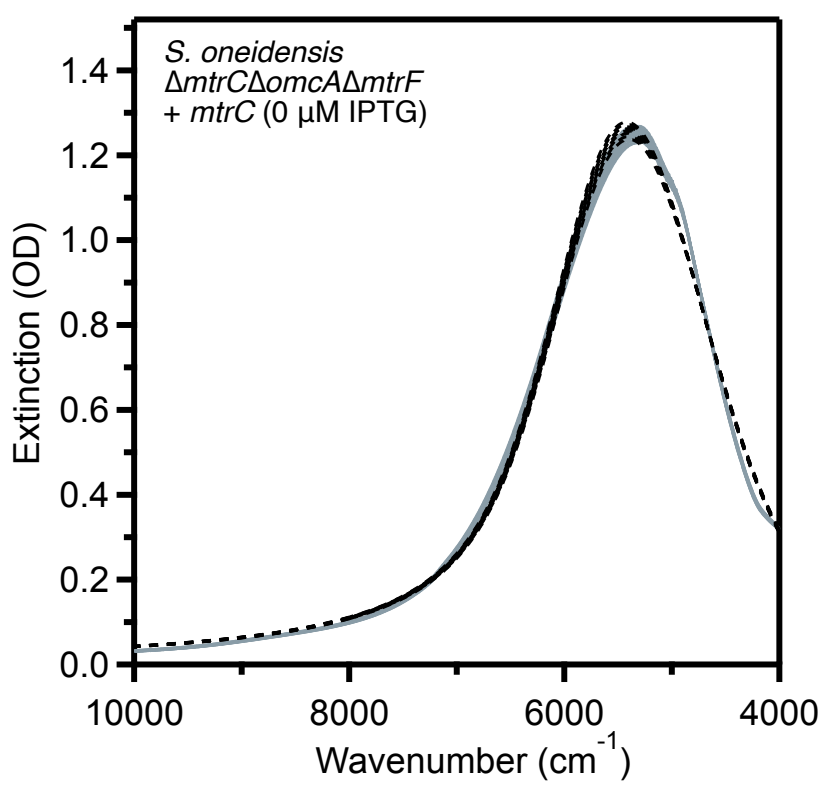

b

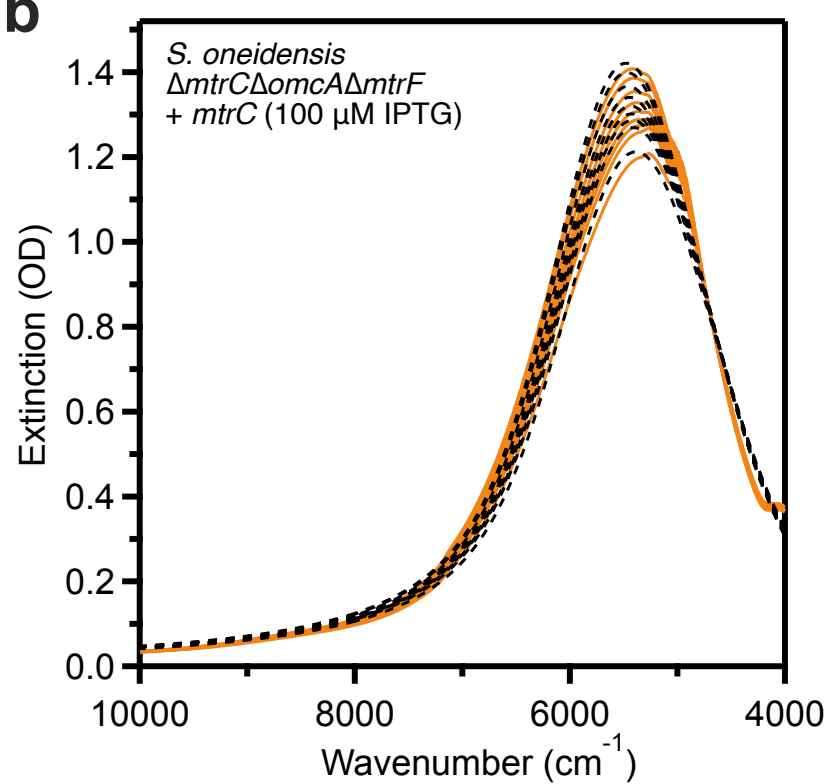

e

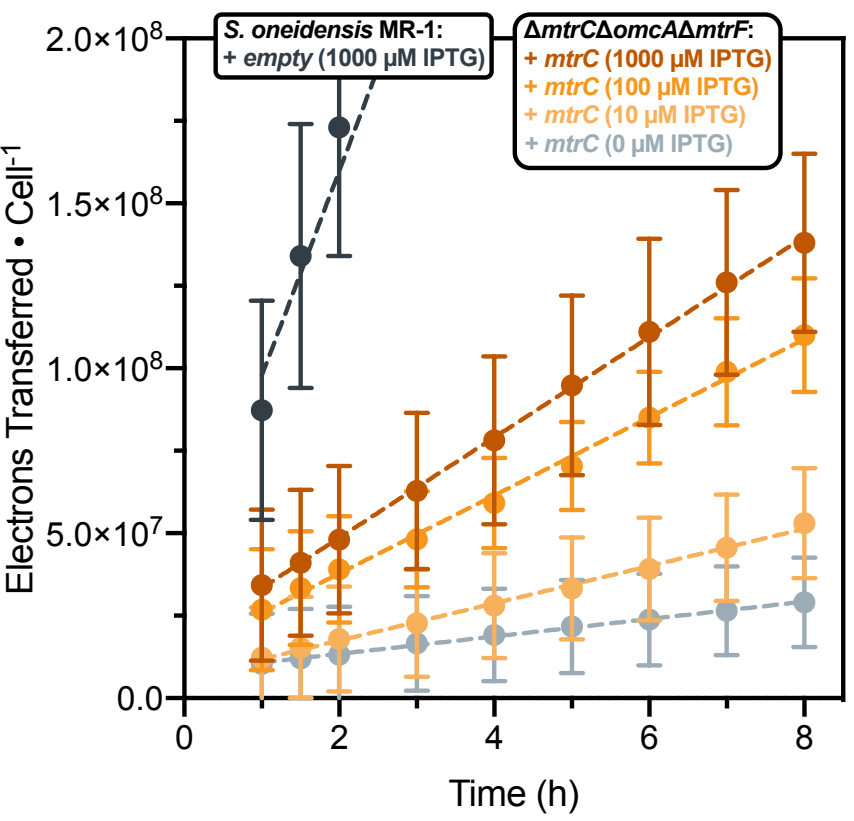

C

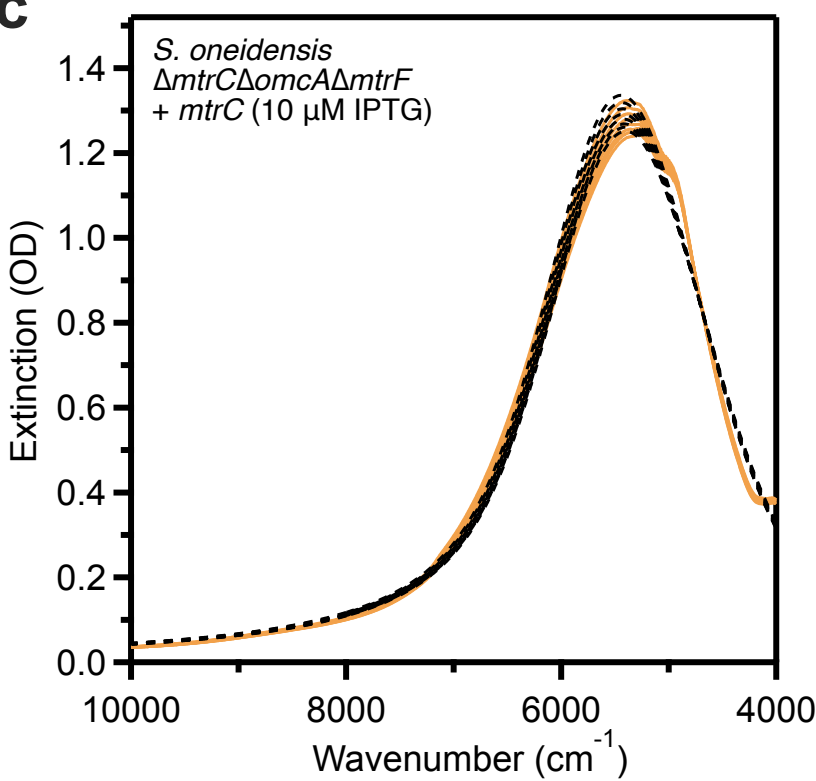

f

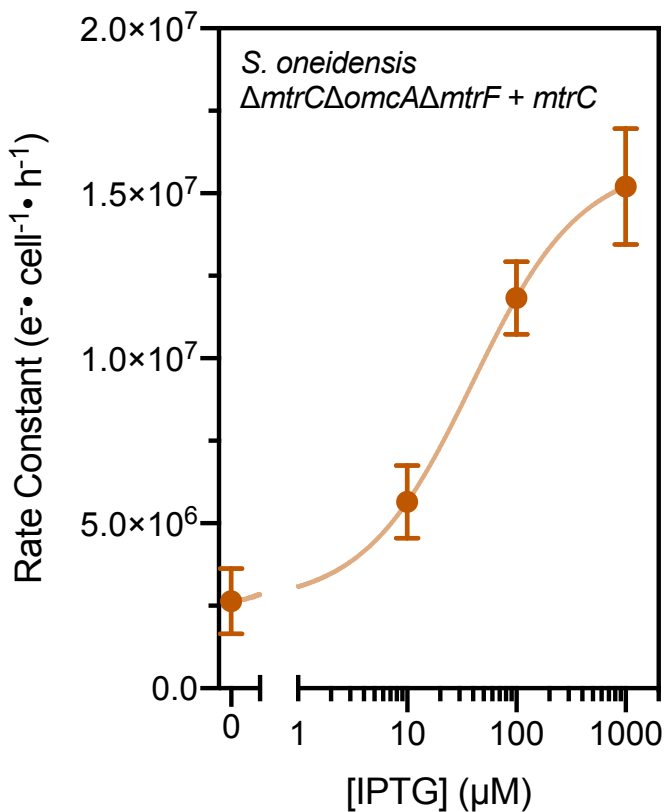

